\title{
Ion irradiation of carbonaceous interstellar analogues
}

\section{Effects of cosmic rays on the $3.4 \mu \mathrm{m}$ interstellar absorption band}

\author{
M. Godard ${ }^{1}$, G. Féraud ${ }^{2}$, M. Chabot ${ }^{3}$, Y. Carpentier ${ }^{2, \star}$, T. Pino ${ }^{2}$, R. Brunetto ${ }^{1}$, J. Duprat ${ }^{4}$, \\ C. Engrand ${ }^{4}$, P. Bréchignac ${ }^{2}$, L. d'Hendecourt ${ }^{1}$, and E. Dartois ${ }^{1}$ \\ ${ }^{1}$ Institut d'Astrophysique Spatiale (IAS, UMR8617), CNRS; Univ Paris-Sud, bât. 121, 91405 Orsay, France \\ e-mail: marie.godard@ias.u-psud.fr \\ 2 Institut des Sciences Moléculaires d'Orsay (ISMO, UMR8214), CNRS; Univ Paris-Sud, bât. 210, 91405 Orsay, France \\ 3 Institut de Physique Nucléaire d'Orsay (IPN, UMR8608), CNRS; Univ Paris-Sud, bât. 102, 91405 Orsay, France \\ ${ }^{4}$ Centre de Spectrométrie Nucléaire et de Spectrométrie de Masse (CSNSM, UMR8609), CNRS; Univ Paris-Sud, bât. 104, \\ 91405 Orsay, France
}

Received 29 November 2010 / Accepted 17 March 2011

\begin{abstract}
Context. A $3.4 \mu \mathrm{m}$ absorption band (around $2900 \mathrm{~cm}^{-1}$ ), assigned to aliphatic C-H stretching modes of hydrogenated amorphous carbons $(\mathrm{a}-\mathrm{C}: \mathrm{H})$, is widely observed in the diffuse interstellar medium, but disappears or is modified in dense clouds. This spectral difference between different phases of the interstellar medium reflects the processing of dust in different environments. Cosmic ray bombardment is one of the interstellar processes that make carbonaceous dust evolve.

Aims. We investigate the effects of cosmic rays on the interstellar $3.4 \mu \mathrm{m}$ absorption band carriers.

Methods. Samples of carbonaceous interstellar analogues ( $\mathrm{a}-\mathrm{C}: \mathrm{H}$ and soot) were irradiated at room temperature by swift ions with energy in the $\mathrm{MeV}$ range (from 0.2 to $160 \mathrm{MeV}$ ). The dehydrogenation and chemical bonding modifications that occurred during irradiation were studied with IR spectroscopy.

Results. For all samples and all ions/energies used, we observed a decrease of the aliphatic C-H absorption bands intensity with the ion fluence. This evolution agrees with a model that describes the hydrogen loss as caused by the molecular recombination of two free $\mathrm{H}$ atoms created by the breaking of $\mathrm{C}-\mathrm{H}$ bonds by the impinging ions. The corresponding destruction cross section and asymptotic hydrogen content are obtained for each experiment and their behaviour over a large range of ion stopping powers are inferred. Using elemental abundances and energy distributions of galactic cosmic rays, we investigated the implications of these results in different astrophysical environments. The results are compared to the processing by UV photons and $\mathrm{H}$ atoms in different regions of the interstellar medium.

Conclusions. The destruction of aliphatic $\mathrm{C}-\mathrm{H}$ bonds by cosmic rays occurs in characteristic times of a few $10^{8}$ years, and it appears that even at longer time scales, cosmic rays alone cannot explain the observed disappearance of this spectral signature in dense regions. In diffuse interstellar medium, the formation by atomic hydrogen prevails over the destruction by UV photons (destruction by cosmic rays is negligible in these regions). Only the cosmic rays can penetrate into dense clouds and process the corresponding dust. However, they are not efficient enough to completely dehydrogenate the $3.4 \mu \mathrm{m}$ carriers during the cloud lifetime. This interstellar component should be destroyed in interfaces between diffuse and dense interstellar regions where photons still penetrate but hydrogen is in molecular form.
\end{abstract}

Key words. cosmic rays - dust, extinction - evolution - methods: laboratory - infrared: ISM - ISM: lines and bands

\section{Introduction}

Interstellar dust continuously interacts with its surrounding environment. Its chemical and physical composition is determined by processes active in space, such as exposure to heat, shocks, $\mathrm{H}$ atoms, UV photons, and cosmic rays. The equilibrium between formation and destruction processes varies within each phase of the interstellar medium (ISM). Laboratory simulations of dust processing in conditions close to those of the interstellar medium may help to understand the evolution of carbonaceous interstellar matter components and to investigate the continuous cycle of organic matter between diffuse and dense regions of the interstellar medium. Infrared spectroscopy is a powerful tool to

* Present address: Laboratory Astrophysics Group of the Max Planck Institute for Astronomy at the Friedrich Schiller University Jena, Institute of Solid State Physics, Helmholtzweg 3, 07743 Jena, Germany. probe the transformations that are undergone in the different environments of the ISM.

The $3.4 \mu \mathrm{m}$ absorption band (around $2900 \mathrm{~cm}^{-1}$ ) is one of the primary carbonaceous dust IR signatures observed in the ISM. This band corresponds to the $\mathrm{C}-\mathrm{H}$ bond vibrations, and in particular to the stretching modes in the methyl $\left(\mathrm{CH}_{3}\right)$ and methylene $\left(\mathrm{CH}_{2}\right)$ groups. It is associated with the corresponding bending modes at $6.85 \mu \mathrm{m}\left(1460 \mathrm{~cm}^{-1}\right)$ and $7.25 \mu \mathrm{m}\left(1380 \mathrm{~cm}^{-1}\right)$. These aliphatic C-H IR absorption bands are widely observed in diffuse interstellar medium in the Milky Way and other galaxies (Wickramasinghe \& Allen 1980; Adamson et al. 1990; Sandford et al. 1991; Pendleton et al. 1994; Whittet et al. 1997; Pendleton \& Allamandola 2002; Spoon et al. 2004; Dartois et al. 2007). Hydrogenated amorphous carbons (a-C:H, also known as HAC in the astrophysical literature) have proven to be good analogues for this aliphatic interstellar dust component through identical IR spectral signatures (Dartois et al. 2005; Chiar et al. 2000). 
Although dust is cycling between the different phases of the ISM and although this aliphatic hydrocarbon component is ubiquitous in diffuse ISM, the only absorption observed in this range in dense clouds is a large featureless band at $3.47 \mu \mathrm{m}$ whose shape is different from the $3.4 \mu \mathrm{m}$ band observed in diffuse ISM. This $3.47 \mu \mathrm{m}$ band correlates with water ice. Its origin and attribution is still debated (Allamandola et al. 1993; Chiar et al. 1996; Brooke et al. 1999; Dartois \& d'Hendecourt 2001; Dartois et al. 2002; Mennella 2010). The recent work of Bottinelli et al. (2010) seems to confirm that this band could coincide with the presence of ammonia hydrate in ice. The widespread distribution of the $3.4 \mu \mathrm{m}$ band in the diffuse interstellar medium and its apparent disappearance or modification in dense clouds give constraints for understanding the formation and evolution of the interstellar aliphatic component. The effects of interstellar processing on this important carbonaceous dust component need to be investigated.

Irradiation by galactic cosmic rays (CR) is one of the interstellar processes known to destroy aliphatic $\mathrm{C}-\mathrm{H}$ bonds, and this induces a decrease of the $3.4 \mu \mathrm{m}$ band intensity. Cosmic rays are composed of highly energetic ions and electrons. Numerous works have been devoted to the study of energetic ions effect on hydrogenated carbonaceous matter by different diagnostic tools such as infrared or Raman spectroscopy, or techniques allowing one to measure the hydrogen content (e.g., Baumann et al. 1987; Prawer et al. 1987; González-Hernández et al. 1988; Fujimoto et al. 1988; Ingram \& McCormick 1988; Zou et al. 1988; Adel et al. 1989; Marée et al. 1996; Pawlak et al. 1997; Som et al. 1999; Baptista et al. 2004; Som et al. 2005; Brunetto et al. 2009). It is observed that the irradiation of these materials results in a decrease of the aliphatic $\mathrm{C}-\mathrm{H}$ spectral signatures and of the hydrogen content. The astrophysical implications of these laboratory simulations of hydrogenated carbon dust have been investigated by Mennella et al. (2003). The experiments were performed using $30 \mathrm{keV} \mathrm{He}$ ions. The effects of other ions and energies (in particular higher energies that are more similar to those of CR) have only been extrapolated. The authors have only considered protons of $1 \mathrm{MeV}$ energy to represent the whole cosmic ray elements and energies (i.e., the approximation of $1 \mathrm{MeV}$ monoenergetic protons). With the present work, we aim at performing a comprehensive study of the effect of cosmic rays on the interstellar aliphatic $\mathrm{C}-\mathrm{H}$ features by combining the results of experiments conducted with a wide range of swift (i.e., energetic) ions and the use of a model describing the effective dehydrogenation of the irradiated material.

We report laboratory experiments aimed at studying energetic ion irradiation of interstellar dust analogues. The different hydrogenated carbonaceous analogue samples are presented in Sect. 2, followed by a description of the irradiation set-up. The experimental results are presented in Sect. 3. They are then implemented in an astrophysical context and discussed in Sect. 4. The conclusions are summarised in Sect. 5.

\section{Experiments}

\subsection{Samples production}

Two different types of analogues of carbonaceous interstellar matter are produced for this study: hydrogenated amorphous carbons (a-C:H or HAC) and carbon soots.

The a-C:H samples were produced at the Institut d'Astrophysique Spatiale (Orsay, France) using a plasmaenhanced chemical vapour deposition (PECVD) system: a microwave-induced plasma of hydrocarbon precursor gas is created in a vacuum chamber (pressure maintained at approximatively $10^{-2}$ mbar) via an Evenson cavity coupled to a $2.45 \mathrm{GHz}$ radio-frequency microwave generator. The precursor gas used is either methane or butadiene. The radicals and ions from the plasma are deposited on a substrate $(\mathrm{KBr}$ or $\mathrm{KCl})$, which is located a few centimetres from the plasma, during a few minutes to approximately one hour depending on the experimental conditions. The few micrometer thick a-C:H film obtained can then be analysed ex situ.

The experiment "Nanograins" of the Institut des Sciences Moléculaires d'Orsay (France) allows us to produce the second kind of carbonaceous materials, hereafter named soot. Incomplete combustion reactions in a fuel-rich, premixed and low pressure (70 mbar) flame nucleate many polycyclic aromatic hydrocarbons and three dimensional soots. For a more detailed description, see Pino et al. (2008). A KBr substrate is placed in front of the flame extraction cone to retrieve the condensable phase. As a result, we obtain a homogenous few micrometer thick sample that can be analysed ex situ.

For both material families, a wide variety of materials can be produced by varying the experimental production conditions (pressure, gas mixture, etc.). Two different types of a-C:H and one type of soot are studied here. The a-C:H type called a-C:H 1 is produced with methane as precursor gas, while a-C:H 2 is synthesised from a plasma of butadiene with addition of argon. Samples of a-C:H 1 and a-C:H 2 are yellow and orange-brown coloured, respectively. The soot is obtained by burning propylene with dioxygen. Soot samples are dark black. In the following, we use a $\mathrm{H} / \mathrm{C}$ ratio of 1.0 and 0.01 , a density of 1.2 and $1.8 \mathrm{~g} \mathrm{~cm}^{-3}$, and a refractive index of 1.4 (Godard \& Dartois 2010) and 1.7 (Bond \& Bergstrom 2006), for a-C:H and soot samples, respectively.

The structure differences between the different kinds of samples (a-C:H 1, a-C:H 2, and soot) result in different IR transmission spectra as seen in Fig. 1. The $\mathrm{C}-\mathrm{H}$ bonds have stretching modes between 2800 and $3100 \mathrm{~cm}^{-1}$, and bending modes between 1300 and $1500 \mathrm{~cm}^{-1}$. The region around $1600 \mathrm{~cm}^{-1}$ corresponds to the $\mathrm{C}=\mathrm{C}\left(\mathrm{sp}^{2}\right)$ stretching modes, that between 1000 and $1500 \mathrm{~cm}^{-1}$ to the $\mathrm{C}-\mathrm{C}\left(\mathrm{sp}^{3}\right)$ stretching motions with some contributions of $\mathrm{sp}^{2} \mathrm{C}=\mathrm{C}$ modes, and that below $1000 \mathrm{~cm}^{-1}$ to the C-H "out-of-plane" bending. Small oxygen contamination (either when the sample is produced or when it is analysed ex situ) can be observed through the $\mathrm{C}=\mathrm{O}$ around $1700 \mathrm{~cm}^{-1}$. The a-C:H 1 samples are highly hydrogenated and they constitute aliphatic materials that reproduce the IR bands observed in the diffuse interstellar medium very well. The type 2 of a-C: $\mathrm{H}$ has a different structure with more olefinic bonds, a higher $\mathrm{sp}^{2} / \mathrm{sp}^{3}$ hybridisation fraction, a lower hydrogen content, and a lower optical gap than type 1 . The two materials also differ by their photoluminescence properties (see a more detailed characterisation of the produced a-C:H in Godard \& Dartois 2010). The soot samples are analogues aiming at reproducing a more aromatic PAH-like carbonaceous phase of interstellar dust. They are far less hydrogenated materials, composed of polyaromatic units linked by aliphatic bridges. The $\mathrm{sp}^{2}$ domains dominate largely the carbon skeleton. As seen in Fig. 1, the soot samples have an absorption continuum owing to electronic transitions, but the a-C:H samples do not.

\subsection{Ion irradiation}

Ion irradiations of these samples were performed at room temperature with different ions and energies at the Tandem accelerator of the Institut de Physique Nucléaire in Orsay (France) 

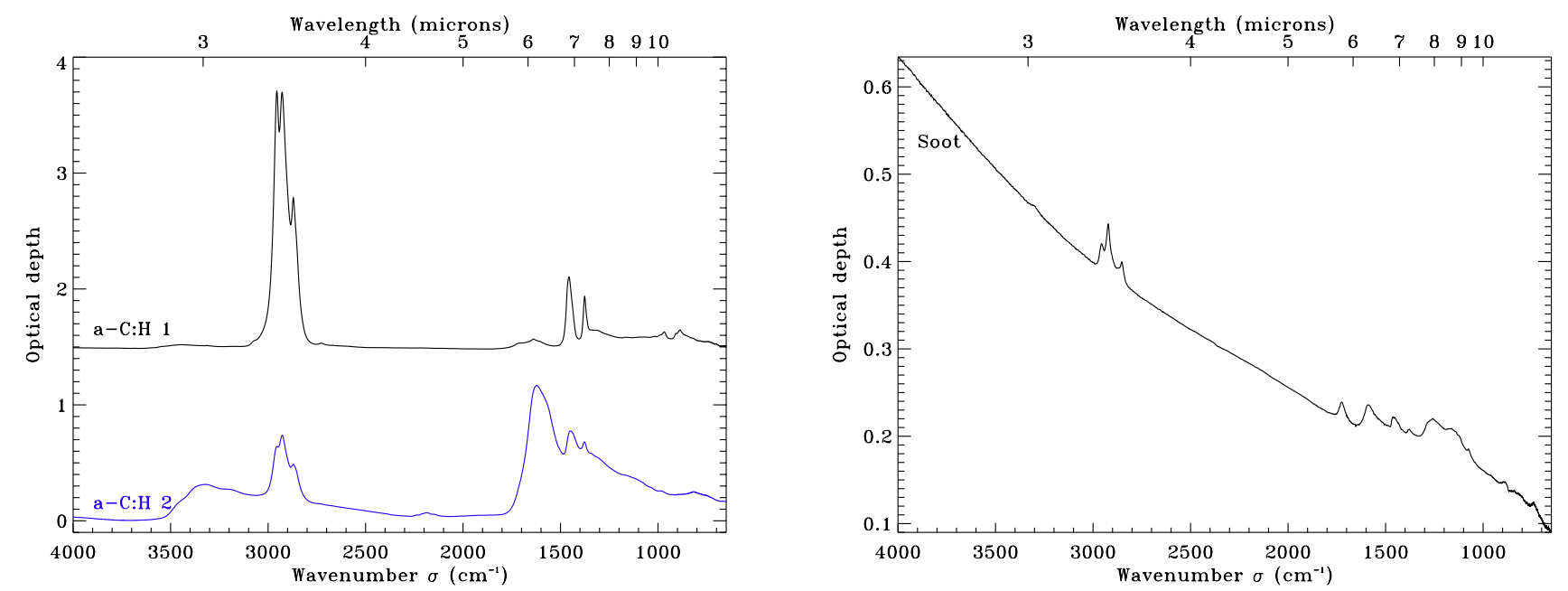

Fig. 1. Infrared spectra (before irradiation) of the different a-C:H and soot samples: IR spectra of samples a-C:H 1 (thickness $d \approx 10 \mu \mathrm{m}$ ) and a-C:H $2(d \approx 7 \mu \mathrm{m})$ are in the left panel and the soot IR spectrum is in the right panel $(d \approx 5 \mu \mathrm{m})$. Spectrum of a-C:H 1 is offset by 1.5 for a better clarity.

in March 2009 (with $10 \mathrm{MeV} \mathrm{H}^{+}, 50 \mathrm{MeV} \mathrm{C}^{5+}, 85 \mathrm{MeV} \mathrm{Si}^{7+}$, and $100 \mathrm{MeV} \mathrm{Ni}^{9+}$ ) and in February 2010 (with $20 \mathrm{MeV} \mathrm{He}^{2+}$, $91 \mathrm{MeV} \mathrm{C}^{6+}$, and $\left.160 \mathrm{MeV} \mathrm{I}^{12+}\right)$. A lower energy irradiation series was conducted at the Laboratory for Experimental Astrophysics (INAF-Catania, Italy) in October 2007 and

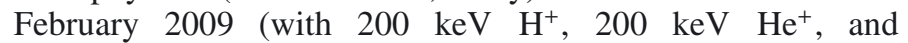
$400 \mathrm{keV} \mathrm{Ar}^{2+}$ ). The samples were irradiated on a diameter of $5 \mathrm{~mm}$ and $1.5 \mathrm{~cm}$ in Orsay and in Catania, respectively.

For the irradiation experiments at the Tandem facility, the samples were placed in a vacuum chamber (few $10^{-7}$ mbar) that faced the ion beam. The IR transmission spectrum of the samples was recorded during irradiation by a Bruker vector 22 FTIR spectrometer, between 6000 and $600 \mathrm{~cm}^{-1}$ with a resolution of $2 \mathrm{~cm}^{-1}$. The acquisition time for one IR transmission spectrum was between 5 and $10 \mathrm{~min}$. The IR beam formed an angle of $45^{\circ}$ with the normal of the sample.

The configuration for the irradiation experiments in Catania was similar, but with the ion and IR beams both at $45^{\circ}$ of the sample surface (Strazzulla et al. 2001). The irradiation occurred at a typical pressure of $10^{-7}$ mbar. The IR spectra were recorded between 8000 and $400 \mathrm{~cm}^{-1}$ with a resolution of $1 \mathrm{~cm}^{-1}$.

The different ions/energies allow us to explore a wide range of stopping cross sections $S=S_{\mathrm{e}}+S_{\mathrm{n}}$, where $S_{\mathrm{e}}$ and $S_{\mathrm{n}}$ are the electronic and nuclear stopping cross section, respectively. The electronic stopping cross section $S_{\mathrm{e}}$ is the electronic stopping power $\mathrm{d} E / \mathrm{d} x$ (i.e. the energy deposited by the ion to the target electrons per unit path length as it moves through the sample) divided by the target density. The electronic stopping power dominates over the nuclear stopping power at high ion speeds (that is the case for cosmic rays). For each experiment, these variables are calculated using the SRIM code (the Stopping and Range of Ions in Matter) (Ziegler et al. 2010) and reported in Table 1.

In this table we also report the ion flux $\Phi_{\mathrm{I}}$ (the ion flux was carefully monitored all along the experiments) and the maximum ion fluence $F_{\mathrm{I} \text {,max }}$ (from $10^{12}$ to $10^{16}$ ions $\mathrm{cm}^{-2}$ ) that was reached within a few hours of irradiation. The corresponding energy flux $\Phi_{\mathrm{E}}$ and maximum deposited energy $F_{\mathrm{E} \text {,max }}$ are calculated by multiplying $\Phi_{\mathrm{I}}$ and $F_{\mathrm{I}, \max }$ by $S_{\mathrm{e}}$. Each sample received from a few $10^{9}$ to a few $10^{12} \mathrm{MeV} \mathrm{mg}^{-1} \mathrm{~s}^{-1}$ and a total energy ranging from $10^{14} \mathrm{MeV} \mathrm{mg}^{-1}$ to $10^{16} \mathrm{MeV} \mathrm{mg}^{-1}$. The flux of ions was between $10^{8}$ and $10^{12}$ ions $\mathrm{cm}^{-2} \mathrm{~s}^{-1}$, but most of these experiments was done with fluxes of a few $10^{10}$ ions $\mathrm{cm}^{-2} \mathrm{~s}^{-1}$.
In the interstellar medium, cosmic ray fluxes are very low ${ }^{1}$. We avoided excessively high fluxes to prevent the heating of the samples. Brunetto et al. (2004) estimate that the temperature increase caused by ion irradiation of diamond samples with fluxes of $10^{13}$ ions $\mathrm{cm}^{-2} \mathrm{~s}^{-1}$ (i.e. at least one order of magnitude higher than those we used) is only $5-10 \mathrm{~K}$. We did not observe different regimes in the reduction of the $\mathrm{C}-\mathrm{H}$ bands when different fluxes were explored for the irradiation of a same sample. During irradiation by $10 \mathrm{MeV} \mathrm{H}$ ions, we did not observe any difference in the hydrogen loss behaviour between fluxes of $10^{11}$ and $10^{12}$ ions $\mathrm{cm}^{-2} \mathrm{~s}^{-1}$. In the experiment with $91 \mathrm{MeV}$ carbon ions, the irradiation of a-C:H 1 sample was stopped for few hours and then restarted without any remarkable discontinuity in the evolution.

Because in the astrophysical context the cosmic ray stopping powers are mostly electronic, we checked that the same is true in the irradiation experiments and that the ions are not implanted in the samples. We thus need to calculate the samples thicknesses $d$. When the interference pattern is visible in the IR spectra, the interfringe $\Delta \sigma$ is measured and the sample thicknesses $d$ are calculated using the formula $d=1 /\left(2 n \Delta \sigma \cos \left(\alpha_{\mathrm{IR}}\right)\right)$, with $n$ the refractive index of the sample, and $\alpha_{\mathrm{IR}}$ the angle of the IR beam incidence with the normal of the sample $\left(\alpha_{\mathrm{IR}}=45^{\circ}\right)$. When this pattern is not visible, the sample thicknesses are estimated from the continuum absorbance for soot, and from the $3.4 \mu \mathrm{m}$ absorbance for a-C:H. The values are given in Table 1 . These estimated thicknesses show that for most of the samples, the ions are implanted in the substrate, located behind the sample (the projected ranges $R_{\mathrm{p}}$ calculated with SRIM, i.e. the length the ion covers in the target material before stopping, are greater than the samples thicknesses), and the irradiation beam can be considered mono-energetic within the sample (i.e. $\Delta E / E \ll 1$, with $\Delta E$ the ion energy difference between each of the two sample surfaces). However, this is not the case anymore for $400 \mathrm{keV} \mathrm{Ar}^{2+}$ ions in a-C:H, and $200 \mathrm{keV} \mathrm{H}{ }^{+}$ions in soot, where the ions are implanted in the sample and the nuclear part of the interaction at the track end becomes substantial. Among the experiments performed in Catania, only the projected range of the $200 \mathrm{keV} \mathrm{H}^{+}$ions in the a-C:H sample is clearly greater than the sample thickness. Therefore, we will not use the results of these

${ }_{1}$ The galactic CR flux is of the order of 20 ions $\mathrm{cm}^{-2} \mathrm{~s}^{-1}$. 


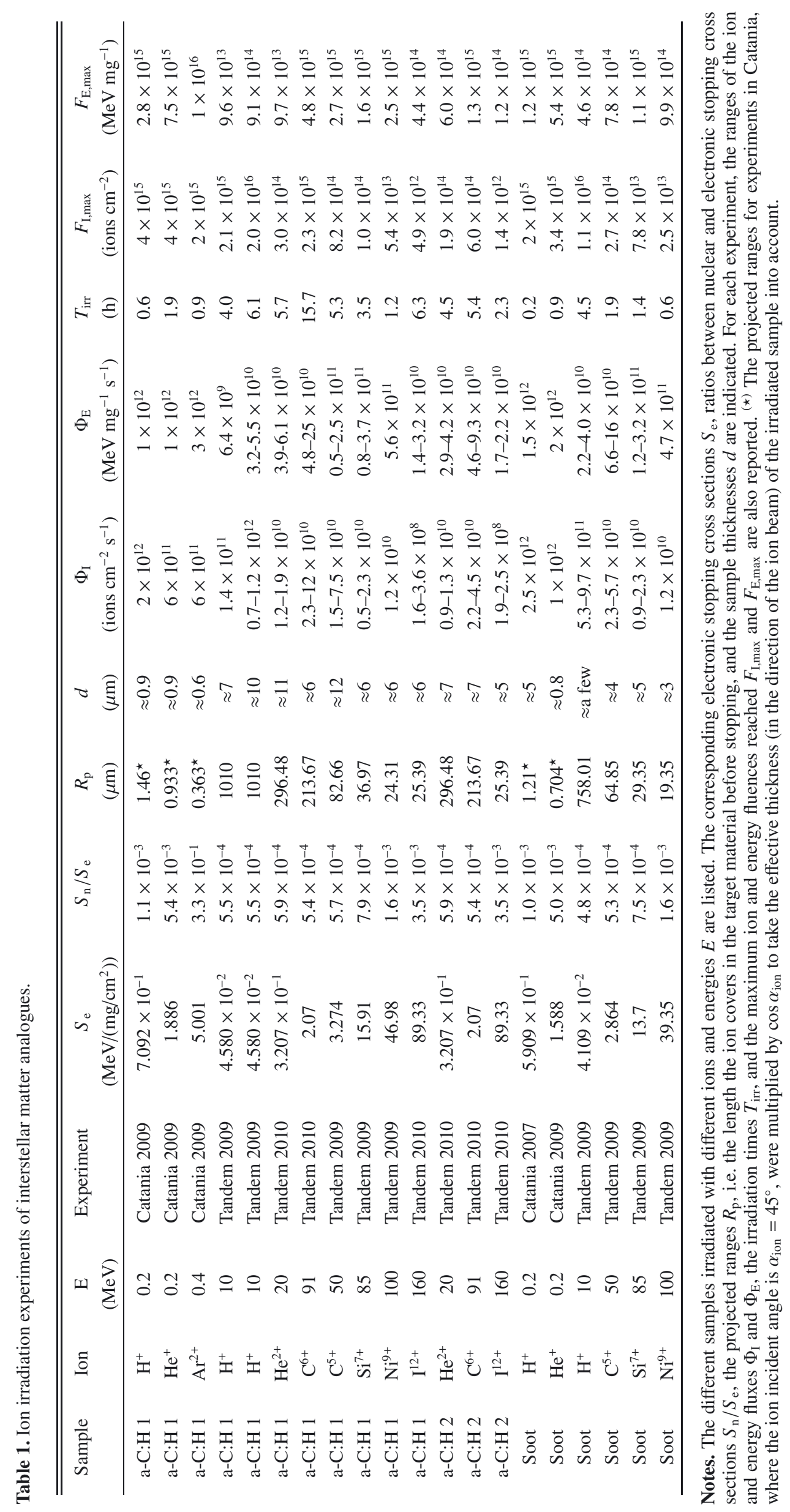




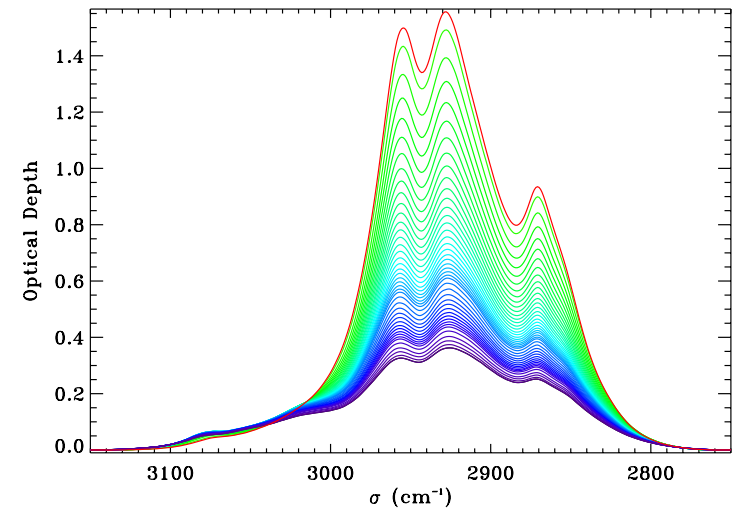

(a) Irradiation of a-C:H 1 with $85 \mathrm{MeV} \mathrm{Si}^{7+}$ between 0 (red) and $1 \times 10^{14}$ ions $\mathrm{cm}^{-2}$ (purple)

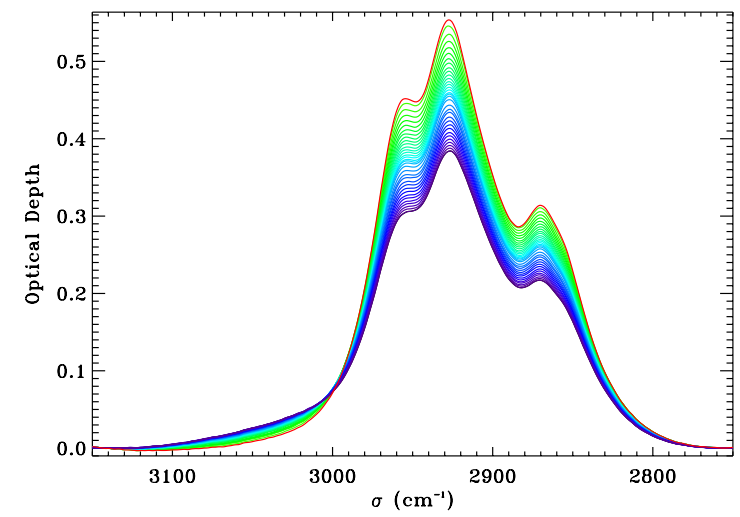

(b) Irradiation of a-C:H 2 with $91 \mathrm{MeV} \mathrm{C}^{6+}$ between 0 (red) and $6 \times 10^{14}$ ions $\mathrm{cm}^{-2}$ (purple)

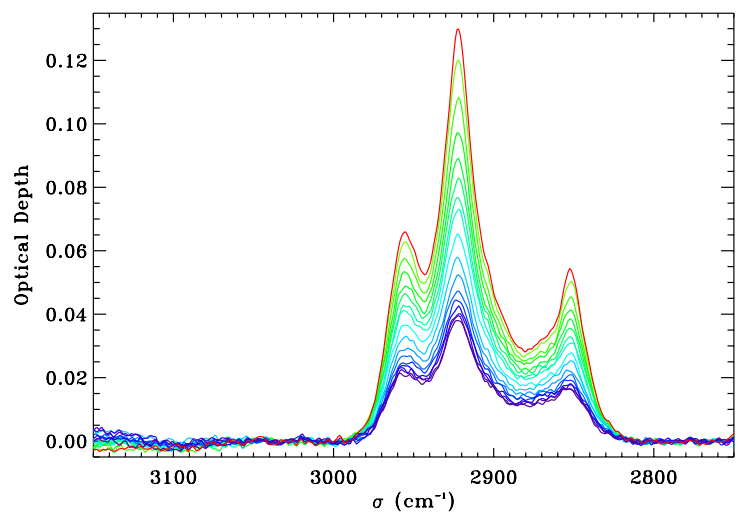

(c) Irradiation of soot with $85 \mathrm{MeV} \mathrm{Si}^{7+}$ between 0 (red) and $8 \times 10^{13}$ ions $\mathrm{cm}^{-2}$ (purple)

Fig. 2. Examples of the destruction of the aliphatic C-H stretching IR absorption feature around $2900 \mathrm{~cm}^{-1}$ during ion irradiation of a$\mathrm{C}: \mathrm{H} 1$ a), a-C:H 2 b), and soot c). The upper (red) line is the initial spectrum, before irradiation. The optical depth decreases (from green to purple lines in the online coloured version) as the fluence rises. The fluence between two spectra is of the order of few $10^{12}$ and few $10^{13}$ ions $\mathrm{cm}^{-2}$ for the $\mathrm{Si}^{7+}$ and $\mathrm{C}^{6+}$ irradiations, respectively.

ions with $R_{\mathrm{p}} / d \leq 1$, which show different behaviours compared to the other irradiation experiments.
We observed no significant modification of the sample thickness (through the interfringe) during irradiation. Therefore, the sputtering that occurs at the sample surface reduces the thickness by only a few percents at most. We do not consider this effect in the following.

\section{Results}

For all carbonaceous samples, the different irradiations result in an effective ${ }^{2}$ destruction of the aliphatic $\mathrm{C}-\mathrm{H}$ component. The optical depths of the $\mathrm{sp}^{3}$ methyl and methylene $\mathrm{C}-\mathrm{H}$ stretching (around $3.4 \mu \mathrm{m}$ or $2900 \mathrm{~cm}^{-1}$ ) and bending modes (6.85 and $7.25 \mu \mathrm{m}, 1460$ and $1380 \mathrm{~cm}^{-1}$ respectively) are decreasing during the irradiation (see Fig. 2). The evolution of the optical depth integrated over the aliphatic C-H stretch band (between 2760 and $3140 \mathrm{~cm}^{-1}$ ) as a function of the deposited energy for different ions/energies is represented in Fig. 3. The evolution of a-C:H 1 (left of Fig. 3) and that of a-C:H 2 (not represented on the figure) for a same ion and energy are very similar (see also Fig. 5).

Other bands appear or disappear with the ion dose as a consequence of the chemical structure modifications induced by ion irradiation. Here, we focus on the dehydrogenation of the material seen through the destruction of aliphatic $\mathrm{C}-\mathrm{H}$ bonding.

\subsection{Determination of the aliphatic $\mathrm{C}-\mathrm{H}$ destruction parameters by ion irradiation: the recombination model}

The integrated optical depth $A=\int \tau \mathrm{d} \sigma$ of the $\mathrm{sp}^{3} \mathrm{C}-\mathrm{H}$ stretching band around $3.4 \mu \mathrm{m}$ (and bending bands around 6.9 and $7.3 \mu \mathrm{m}$ ) decreases with the ion fluence, and thus with the deposited energy (Fig. 3). This evolution can be fitted by a function of the form

$A=\frac{1}{1 / A_{\mathrm{f}}+\left(1 / A_{\mathrm{i}}-1 / A_{\mathrm{f}}\right) \exp \left(-\sigma_{\mathrm{d}} F_{\mathrm{I}}\right)}$,

where $F_{\mathrm{I}}$ is the ionic fluence (ion $\mathrm{cm}^{-2}$ ), and $\sigma_{\mathrm{d}}$ the effective C-H destruction cross section $\left(\mathrm{cm}^{2}\right.$ ion $\left.^{-1}\right) . A_{\mathrm{i}}$ and $A_{\mathrm{f}}$ are the initial and asymptotic value at an infinite dose of the integrated optical depth, respectively. The value of $A$ is assumed to be proportional to the hydrogen concentration $\rho$. The Eq. (1) results then of the equation

$\rho=\frac{1}{1 / \rho_{\mathrm{f}}+\left(1 / \rho_{\mathrm{i}}-1 / \rho_{\mathrm{f}}\right) \exp \left(-\sigma_{\mathrm{d}} F_{\mathrm{I}}\right)}$,

where $\rho_{\mathrm{i}}$ and $\rho_{\mathrm{f}}$ are the initial and asymptotic value of the hydrogen concentration.

This function, which describes the hydrogen content evolution with ion fluence, results from a model developed by Adel et al. (1989) and Marée et al. (1996). This model is based on the fact that hydrogen leaves the a-C:H films in molecular form (Wild \& Koidl 1987; Möller \& Scherzer 1987). Therefore, the hydrogen evolution is based on a second order kinetic process. In this approach, the recombination of two hydrogen atoms into molecular hydrogen, which results from the breaking of two $\mathrm{C}-\mathrm{H}$ bonds by the electronic energy deposition of a passing ion occurs in the bulk of the irradiated material. The resulting $\mathrm{H}_{2}$ molecule then rapidly diffuses to the surface and is lost from the material without further interactions. When a C-H bond is broken, the hydrogen atom diffuses within a characteristic distance $l$ in the ion track before it is either trapped by a reactive

2 As explained in Sect. 3.1, C-H bonds are broken by impinging ions, but some of the free $\mathrm{H}$ atoms form new bonds in the material. 

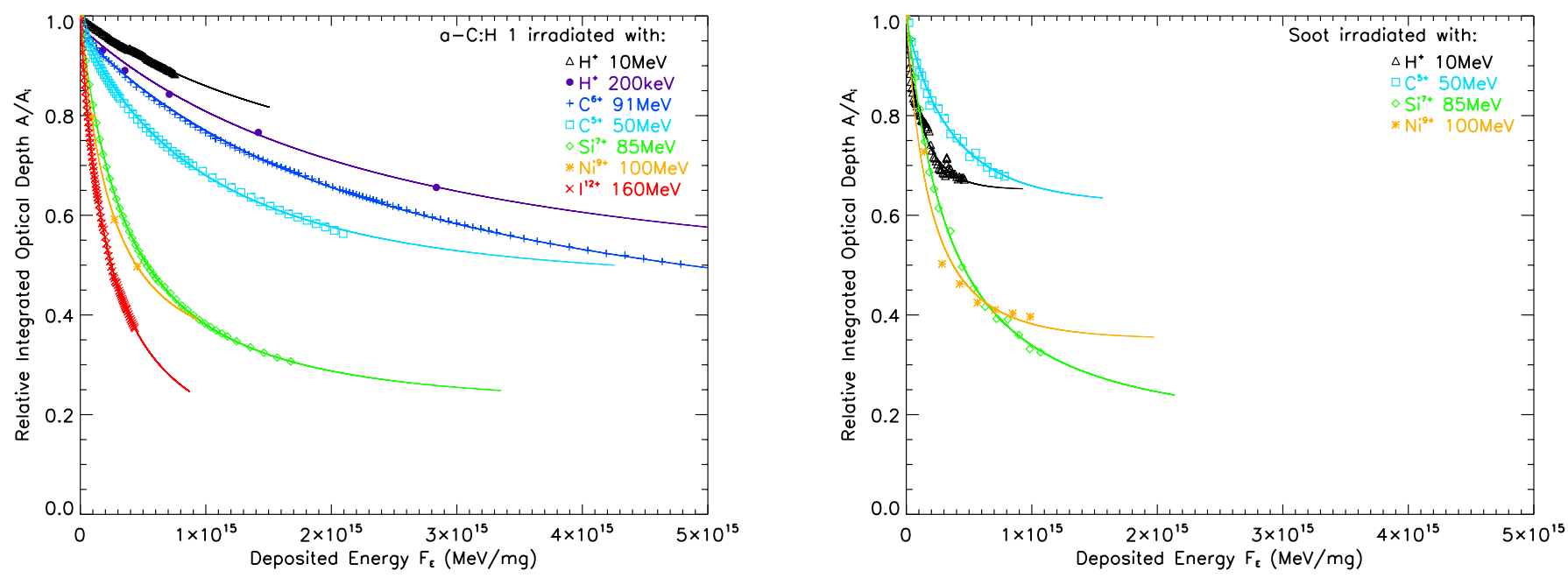

Fig. 3. Examples of evolution of the relative integrated optical depth of the aliphatic C-H stretching band with the energy deposited by the ions and the associated fits (recombination model). The deposited energy is calculated from the ion fluence: $F_{\mathrm{E}}=F_{\mathrm{I}} \cdot S_{\mathrm{e}}$. The a-C:H and soot samples are represented on the left and on the right, respectively.

site in the irradiated material, or is recombined with another free $\mathrm{H}$ atom. This distance defines a recombination volume $V$, inside the track of radius $r$, in which two free hydrogen atoms that are released by the energy deposition of the same ion can recombine as a $\mathrm{H}_{2}$ molecule. The probability of breaking a $\mathrm{C}-\mathrm{H}$ bond with one ion is $P_{\text {break }}$ and the probability of molecular recombination of two $\mathrm{H}$ atoms diffusing in the volume $V$ is $P_{\text {rec }}$. The number of possible pairs of $\mathrm{H}$ atoms that can be formed by one ion in the volume $V$ is $\frac{1}{2} \cdot \rho V P_{\text {break }} \cdot(\rho V-1) P_{\text {break. The number of }}$ $\mathrm{H}$ atoms lost as $\mathrm{H}_{2}$ molecules per energetic ion in the volume $V$ is thus $\rho V(\rho V-1) P_{\text {break }}^{2} P_{\text {rec }}$. The differential equation that describes the hydrogen depletion of the film with the ion fluence $F_{\mathrm{I}}$ is given by

$\mathrm{d} \rho=-\rho(\rho V-1) P_{\text {break }}^{2} P_{\text {rec }} \cdot \pi r^{2} \mathrm{~d} F_{\mathrm{I}}$.

Equation (2) is the solution for this differential equation with $\sigma_{\mathrm{d}}=P_{\text {break }}^{2} P_{\text {rec }} \pi r^{2}$ and $\rho_{\mathrm{f}}=1 / V$. When only one hydrogen atom remains in the recombination volume, then $\rho V=1$ and the $\mathrm{H}$ release via hydrogen molecular recombination ceases or becomes very improbable. The latter explains the link between $V$ and the final hydrogen density $\rho_{\mathrm{f}}$. Equation (3) can be written as

$\frac{\mathrm{d} \rho}{\mathrm{d} F_{\mathrm{I}}}=-\frac{\sigma_{\mathrm{d}}}{\rho_{\mathrm{f}}} \rho^{2}\left(1-\frac{\rho_{\mathrm{f}}}{\rho}\right)$

Equation (4) is valid for an irradiation perpendicular to the sample surface. Otherwise, the angle of incidence should be taken into account in the section of the ion track. In the irradiation performed in Catania, the ion beam has a direction of $45^{\circ}$ with respect to the sample surface, $\sigma_{\mathrm{d}}$ in previous equations becomes $\frac{\sigma_{\mathrm{d}}}{\sin 45}$.

This model can explain the hydrogen depletion induced by ion irradiations in hydrogenated amorphous carbon films. It has been applied for such materials by Adel et al. (1989), Marée et al. (1996), Som et al. (1999), and Baptista et al. (2004), and also to other organic layers (e.g., in Adel et al. 1989; Marée et al. 1996; and Baptista et al. 2004).

We applied to our data fits corresponding to the recombination model, based on Eq. (2), using $\sigma_{\mathrm{d}}$ and $\rho_{\mathrm{f}}$ as adjustable parameters. The best-fit parameters and the associated errors are determined by a $\chi^{2}$ minimisation. The obtained destruction cross section $\sigma_{\mathrm{d}}$, relative final hydrogen content $A_{\mathrm{f}} / A_{\mathrm{i}}$, and corresponding recombination volume $V=1 / \rho_{\mathrm{f}}$ values for the different experiments are reported in Table 2 (for explanation about the estimation of $V$, see Sect. 3.3).

Alternatively, the hydrogen concentration decrease can also be fitted assuming a first-order kinetic process (Mennella et al. 2003; Pawlak et al. 1997), with an exponential function and an added constant representing the aliphatic feature residual intensity at high ion fluences. This fit is a simple phenomenological function. In the Appendix, we report the results obtained with this exponential fit and show that Eq. (2), which corresponds to the recombination model, fits our data better.

\subsection{Destruction cross section}

We represent in Fig. 4 the different values of $\sigma_{\mathrm{d}}$ as a function of the electronic stopping cross section $S_{\mathrm{e}}$ for the a-C:H and soot irradiation experiments. For a-C:H, the destruction cross section estimated from the aliphatic $\mathrm{C}-\mathrm{H}$ bending bands are also plotted. These $\sigma_{\mathrm{d}}$ are, as expected, very similar to those determined through the stretching band. The fairly large error bars illustrate the strong dependence of $\sigma_{\mathrm{d}}$ on $A_{\mathrm{f}}$ with Eq. (1). Effectively, the values of $A_{\mathrm{f}}$ are not always well constrained in our data because we lack experimental points at higher fluence. These uncertainties largely depend on the asymptotic behaviour, which requires high ion fluence. The latter is strongly limited by the instantaneous fluxes that should not induce heating of the sample, and by the available time on the ion irradiation facility. The irradiation by $20 \mathrm{MeV}$ helium ions $\left(S_{\mathrm{e}} \approx 0.2 \mathrm{MeV} /\left(\mathrm{mg} / \mathrm{cm}^{2}\right)\right)$ sets only an upper limit on the destruction cross section because a higher fluence is required. For low $S_{\mathrm{e}}$ experiments, this high fluence was difficult to reach without obtaining excessively high ion fluxes.

\subsubsection{Influence of the material irradiated}

a-C:H 1 and a-C:H 2 have similar values of $\sigma_{\mathrm{d}}$. It appears that, although the carbon skeleton and the hydrogen density of these two materials are slightly different, this does not have a strong influence on the destruction cross sections within the explored range. In the case of soots, the $\sigma_{\mathrm{d}}$ values are also comparable to those of a-C:H, within the experimental uncertainties (higher 
Table 2. Results of ion irradiation experiments obtained with the recombination model fits.

\begin{tabular}{|c|c|c|c|c|c|c|}
\hline Sample & Ion & $\begin{array}{c}E \\
(\mathrm{MeV})\end{array}$ & $\begin{array}{c}S_{\mathrm{e}} \\
\left(\mathrm{MeV} /\left(\mathrm{mg} / \mathrm{cm}^{2}\right)\right)\end{array}$ & $\begin{array}{c}\sigma_{\mathrm{d}} \\
\left(\mathrm{cm}^{2}\right)\end{array}$ & $A_{\mathrm{f}} / A_{\mathrm{i}}$ & $\begin{array}{c}V \\
\left(\AA^{3}\right)\end{array}$ \\
\hline $\mathrm{a}-\mathrm{C}: \mathrm{H} 1$ & $\mathrm{H}^{+}$ & 10 & $4.580 \times 10^{-2}$ & $<3.5 \times 10^{-16}$ & - & - \\
\hline $\mathrm{a}-\mathrm{C}: \mathrm{H} 1$ & $\mathrm{H}^{+}$ & 10 & $4.580 \times 10^{-2}$ & $(0.3) 1.9(5.6) \times 10^{-17}$ & $(0.02) 0.66(0.82)$ & $>22$ \\
\hline $\mathrm{a}-\mathrm{C}: \mathrm{H} 1$ & $\mathrm{He}^{2+}$ & 20 & $3.207 \times 10^{-1}$ & $<5.3 \times 10^{-15}$ & - & - \\
\hline $\mathrm{a}-\mathrm{C}: \mathrm{H} 1$ & $\mathrm{H}^{+}$ & 0.2 & $7.092 \times 10^{-1}$ & $(0.3) 1.2(3.6) \times 10^{-16}$ & $(0.01) 0.50(0.62)$ & $>29$ \\
\hline $\mathrm{a}-\mathrm{C}: \mathrm{H} 1$ & $\mathrm{C}^{6+}$ & 91 & 2.07 & $(0.7) 3.2(7.8) \times 10^{-16}$ & $(0.17) 0.35(0.45)$ & (40)51(104) \\
\hline $\mathrm{a}-\mathrm{C}: \mathrm{H} 1$ & $\mathrm{C}^{5+}$ & 50 & 3.274 & $(0.3) 1.7(3.9) \times 10^{-15}$ & $(0.23) 0.47(0.55)$ & $(32) 38(78)$ \\
\hline $\mathrm{a}-\mathrm{C}: \mathrm{H} 1$ & $\mathrm{Si}^{7+}$ & 85 & 15.91 & $(0.6) 1.0(1.5) \times 10^{-14}$ & $(0.21) 0.23(0.25)$ & (73)79(83) \\
\hline $\mathrm{a}-\mathrm{C}: \mathrm{H} 1$ & $\mathrm{Ni}^{9+}$ & 100 & 46.98 & $(1.2) 6.8(15) \times 10^{-14}$ & $<0.37$ & $>49$ \\
\hline $\mathrm{a}-\mathrm{C}: \mathrm{H} 1$ & $\mathrm{I}^{12+}$ & 160 & 89.33 & $(0.3) 4.1(12) \times 10^{-14}$ & $(0.01) 0.10(0.18)$ & $(98) 184(1470)$ \\
\hline $\mathrm{a}-\mathrm{C}: \mathrm{H} 2$ & $\mathrm{He}^{2+}$ & 20 & $3.207 \times 10^{-1}$ & $<1.0 \times 10^{-17}$ & ${ }^{-}$ & - \\
\hline $\mathrm{a}-\mathrm{C}: \mathrm{H} 2$ & $\mathrm{C}^{6+}$ & 91 & 2.07 & $(4.0) 8.8(16) \times 10^{-16}$ & $(0.46) 0.55(0.61)$ & (29)33(38) \\
\hline $\mathrm{a}-\mathrm{C}: \mathrm{H} 2$ & $\mathrm{I}^{12+}$ & 160 & 89.33 & $(0.02) 1.8(12) \times 10^{-13}$ & $(0.01) 0.38(0.68)$ & $(26) 47(1220)$ \\
\hline Soot & $\mathrm{H}^{+}$ & 10 & $4.109 \times 10^{-2}$ & $(0.001) 2.2(6.1) \times 10^{-16}$ & $(0.00) 0.65(0.67)$ & $>27$ \\
\hline Soot & $\mathrm{C}^{5+}$ & 50 & 2.864 & $(0.008) 5.5(14) \times 10^{-15}$ & $(0.00) 0.62(0.68)$ & $>26$ \\
\hline Soot & $\mathrm{Si}^{7+}$ & 85 & 13.7 & $(5.9) 7.8(30) \times 10^{-15}$ & $(0.00) 0.18(0.32)$ & $>55$ \\
\hline Soot & $\mathrm{Ni}^{9+}$ & 100 & 39.35 & $(0.02) 8.3(23) \times 10^{-14}$ & $(0.00) 0.35(0.40)$ & $>45$ \\
\hline
\end{tabular}

Notes. The aliphatic C-H destruction cross section $\sigma_{\mathrm{d}}$, the relative asymptotic optical depth $A_{\mathrm{f}} / A_{\mathrm{i}}$, and the recombination volume $V$ are given (see text for explanation). These values are determined from the $\mathrm{C}-\mathrm{H}$ stretching modes at $3.4 \mu \mathrm{m}$. The extreme values of each parameters are given between parenthesis.

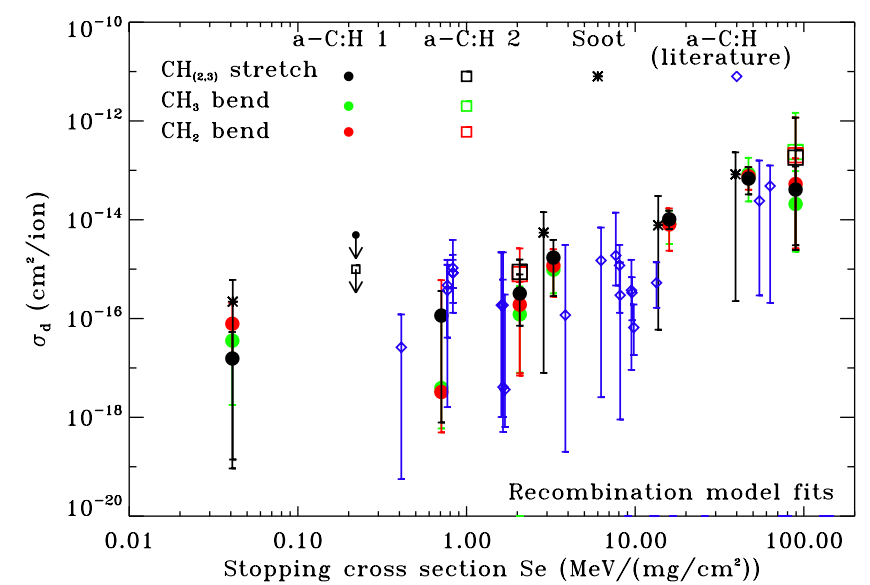

Fig. 4. Aliphatic C-H destruction cross section $\sigma_{\mathrm{d}}$ obtained with the recombination model as a function of the electronic cross section $S_{\mathrm{e}}$ for the different ion/energy irradiation of our a-C:H (filled dots for a-C:H 1 , and squares for a-C:H 2) and soot (black asterisks) samples. Cross sections calculated from data of different a-C:H irradiation studies are represented with blue diamonds (Adel et al. 1989; Baptista et al. 2004; Baumann et al. 1987; Fujimoto et al. 1988; González-Hernández et al. 1988; Ingram \& McCormick 1988; Marée et al. 1996; Mennella et al. 2003; Pawlak et al. 1997; Prawer et al. 1987; Som et al. 2005, 1999; Zou et al. 1988). See text for details.

for soot than a-C:H). This similarity seems surprising because of the large difference in the $\mathrm{H} / \mathrm{C}$ ratio, for instance. Soot and a-C:H strongly differ in their carbon skeleton and hydrogen density, the soot being mainly composed of polyaromatic units, poorly organised, and cross-linked by aliphatic bridges. It implies that the aliphatic domains are localised at the edge of the aromatic domains, and that the (aliphatic) hydrogen density is far from being homogeneous within the material. Thus, as long as the recombination volume is smaller than or comparable to the aliphatic domain extent, the hydrogen experiences an environment similar as in a-C:H. It may also indicate that this ionic irradiation does not create efficient reactive sites in the aromatic domains as suggested by the infrared spectra of the irradiated soot. These findings show that only the local environments experienced by the hydrogen atoms, which is identified as the recombination volume, determined the effective destruction cross section.

In Fig. 4 we also plotted the effective destruction cross section (seen through the aliphatic $\mathrm{C}-\mathrm{H}$ stretch band) of previous a-C:H irradiation experiments (Adel et al. 1989; Baptista et al. 2004; Baumann et al. 1987; Fujimoto et al. 1988; González-Hernández et al. 1988; Ingram \& McCormick 1988; Marée et al. 1996; Mennella et al. 2003; Pawlak et al. 1997; Prawer et al. 1987; Som et al. 2005, 1999; Zou et al. 1988). These cross sections were deduced from the available data and using Eq. (1). We did not represent in this figure the experiments where the nuclear cross section plays a role $\left(S_{\mathrm{n}} / S_{\mathrm{e}}>0.5\right)$, or where implantation in the sample occurs $\left(d / R_{\mathrm{p}}>0.9\right)$ in order to compare experiments pertaining to the same irradiation conditions. Even if it is difficult to compare results from different experiments, our results and those from previous works are, for most of them, on the same order of magnitude and show the same behaviour with $S_{\mathrm{e}}$. These materials span a wide range of hydrogen atomic concentration, from few to about fifty percents. Therefore, the measured destruction cross sections for aliphatic $\mathrm{C}-\mathrm{H}$ bonds seem weakly dependent on the irradiated carbonaceous material and may be used as a general characteristic.

Below we will use only our results on a-C:H 1 because they were better explored and thus have lower uncertainties.

\subsubsection{Dependence on the stopping power}

The aliphatic $\mathrm{C}-\mathrm{H}$ destruction cross section seems to vary as a power law of $S_{\mathrm{e}}$. We fitted the results with:

$\sigma_{\mathrm{d}}=K \cdot S_{\mathrm{e}}^{\alpha}$.

The best and extreme fits for our a-C:H results obtained with the recombination model are shown in Fig. 5. The parameters of this fit for a-C:H and soot are given in Table 3 (for $S_{\mathrm{e}}$ in $\mathrm{MeV} /\left(\mathrm{mg} / \mathrm{cm}^{2}\right)$ and $\sigma_{\mathrm{d}}$ in $\left.\mathrm{cm}^{2}\right)$. 


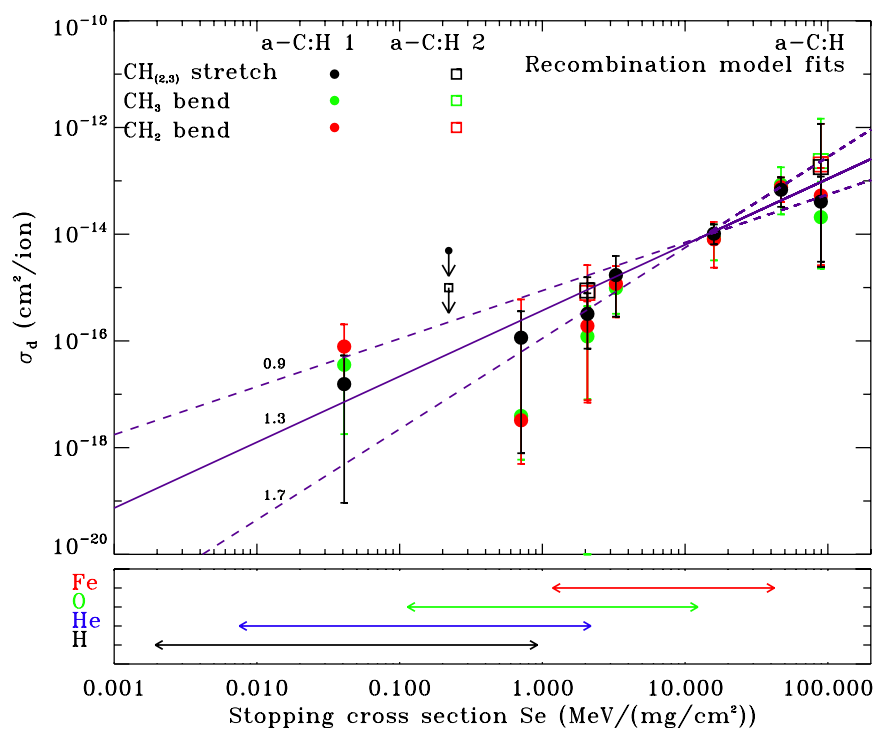

Fig. 5. Aliphatic C-H destruction cross section $\sigma_{\mathrm{d}}$ as function of the electronic cross section $S_{\mathrm{e}}$ for the different ion/energy irradiation of the a-C:H samples. The values corresponding to the $\mathrm{C}-\mathrm{H}$ stretching modes at $3.4 \mu \mathrm{m}$ (in black), the $\mathrm{CH}_{2}$ bending mode at $6.85 \mu \mathrm{m}$ (in red), and the $\mathrm{CH}_{3}$ bending mode at $7.25 \mu \mathrm{m}$ (in green) are represented for both a-C:H 1 (filled dots) and a-C:H 2 (squares). The best fit and the fits with extreme values of $\alpha$ are plotted (the corresponding values of $\alpha$ are written). The coloured arrows represent the $S_{\mathrm{e}}$ range of the few major cosmic ray elements.

Table 3. Values of the $\sigma_{\mathrm{d}}$ fit parameters from Eq. (5).

\begin{tabular}{ccc}
\hline \hline Material & $\alpha$ & $K$ \\
\hline a-C $: \mathrm{H}$ & $1.3( \pm 0.4)$ & $5( \pm 4) \times 10^{-16}$ \\
Soot & $1.0( \pm 0.5)$ & $2.2( \pm 1.2) \times 10^{-15}$ \\
\hline
\end{tabular}

Notes. $K$ is given in $\mathrm{cm}^{2-2 \alpha} \mathrm{MeV}^{-\alpha} \mathrm{mg}^{\alpha}$ units. $\sigma_{\mathrm{d}}$ are obtained from the recombination model.

The value of $\alpha$ is not completely determined and lies between 0.5 and 1.7 , but the values matching the a-C:H and soot data are between 0.9 and 1.5. In the following, we will use $\alpha$ values of 1.0, 1.2 and 1.4 to show the consequences of different $\sigma_{\mathrm{d}}$ behaviours. Mennella et al. (2003) assume a power of 1 (i.e. proportionality between $\sigma_{\mathrm{d}}$ and $S_{\mathrm{e}}$ ), while Baptista et al. (2004) found a power of approximately 2. The model of Marée et al. (1996) not only explains the hydrogen release as a second-order kinetic process, but also explains the dependence of the release rate on the ion stopping power in the material. As they explained, the stopping power appears to be proportional to $r^{2}$. We saw above that the recombination model predicts values of $\sigma_{\mathrm{d}}$ that are also proportional to $r^{2}$. Thus, in the description given by the model developed by Marée et al. (1996), $\sigma_{\mathrm{d}}$ should be proportional to $S_{\mathrm{e}}$ (i.e., $\alpha=1$ ). This is compatible with our experimental results.

To compare the stopping cross sections $S_{\mathrm{e}}$ explored by our experiments with the $S_{\mathrm{e}}$ of cosmic rays, we represented in Figs. 5 and 6 the $S_{\text {e }}$ range of a few major cosmic ray elements (coloured arrows in the bottom plots). These ranges are determined for each element, from the $S_{\mathrm{e}}$ distribution as a function of the energy (calculated with the SRIM program for each projectile, for $\mathrm{H} / \mathrm{C}$ ratio and density corresponding to our a-C:H target material) and the cosmic ray distribution of energy. The

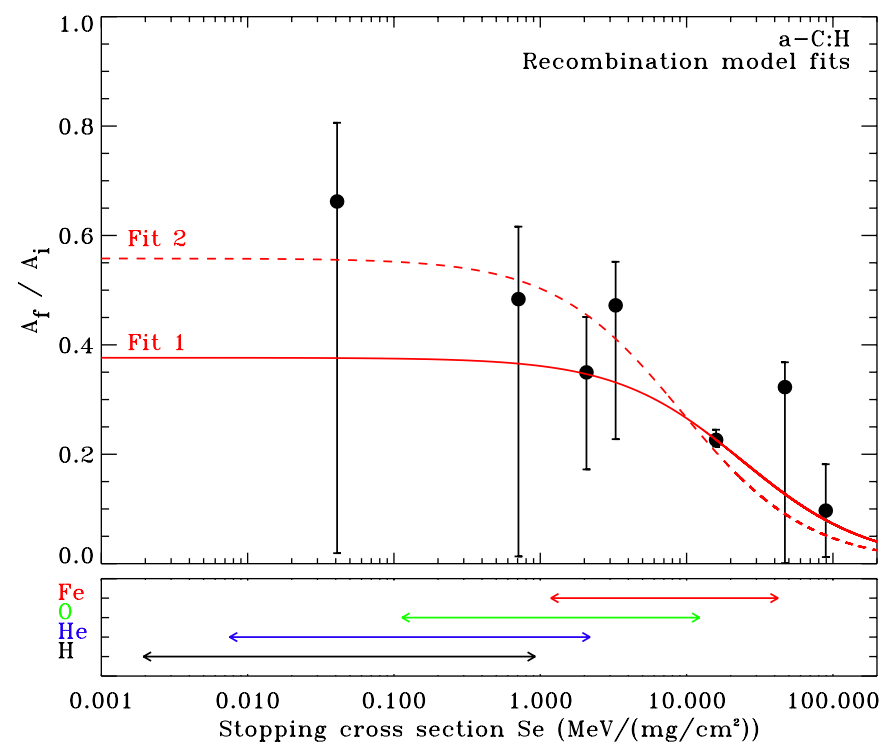

Fig. 6. Final relative integrated optical depth $A_{\mathrm{f}} / A_{\mathrm{i}}$ of $\mathrm{C}-\mathrm{H}$ stretching modes, obtained after infinite irradiation as function of the electronic cross section $S_{\mathrm{e}}$ for the different irradiation ion/energy of a-C:H 1 samples. Two different fits corresponding to the linear increase of the recombination volume $V$ with $S_{\mathrm{e}}$ (Eq. (6)) are plotted: the solid line (fit 1) is the best fit, and the dashed line (fit 2) represents an extreme fit for which the asymptotic $A_{\mathrm{f}} / A_{\mathrm{i}}$ are the highest for low $S_{\mathrm{e}}$. The coloured arrows represent the $S_{\mathrm{e}}$ range of the few major cosmic ray elements.

CR energy from $100 \mathrm{eV} /$ nucleon up to $10 \mathrm{GeV} /$ nucleon are considered because, below and above this energy range, the CR flux becomes either too low and/or the induced destruction is inefficient (cf. Sect. 4.1 and Fig. 8).

\subsection{Residual $H$ content and recombination volume}

We found that the asymptotic values at infinite fluence of the integrated optical depth of the aliphatic $\mathrm{C}-\mathrm{H}$ bands is not zero, and thus a fraction of the initial hydrogen content remains in the irradiated material. This residual $\mathrm{H}$ content is reached when the $\mathrm{C}-\mathrm{H}$ bond concentration is low enough so that the free $\mathrm{H}$ atoms liberated by an ion are too far from each other to recombine (i.e. the mean distance between $\mathrm{H}$ atoms in the material is greater than the radius defined by the recombination volume), and cannot form $\mathrm{H}_{2}$ molecules. The hydrogen is captured by the material and does not escape. In Fig. 6 the parameters $A_{\mathrm{f}} / A_{\mathrm{i}}$ obtained from the recombination model fits of a-C:H are represented as a function of the electronic stopping cross section $S_{\mathrm{e}}$. The final hydrogen content decreases when the stopping cross section increases. For the experiments with the lowest stopping cross section (i.e. those with $S_{\mathrm{e}}<1 \mathrm{MeV} /\left(\mathrm{mg} / \mathrm{cm}^{2}\right)$ ), the value of $A_{\mathrm{f}}$ cannot be well determined and only upper limits are obtained.

Assuming an initial hydrogen volumic concentration $\rho_{\mathrm{i}}$ around $5.6 \times 10^{22} \mathrm{H}$ at. $\mathrm{cm}^{-3}$ for a-C:H (estimated from the values of density and $\mathrm{H} / \mathrm{C}$ ratio given in Sect. 2.1), we obtain $\rho_{\mathrm{f}}$ from $A_{\mathrm{f}} / A_{\mathrm{i}}$. In the recombination model, the recombination volume $V$ is the inverse of the hydrogen concentration $\rho_{\mathrm{f}}$. The estimated values of $V$ are given in Table 2. With the same method, we obtain an estimated mean hydrogen density for soot of $9.0 \times$ $10^{20} \mathrm{H}$ at. $\mathrm{cm}^{-3}$. However, contrary to a-C:H, the aliphatic hydrogen density is not homogeneous in soot, and is higher in the aliphatic bridges that link the aromatic units. The $V$ estimates for soot given in Table 2 are thus calculated with a local hydrogen 
density equal to the a-C:H $\rho_{\mathrm{i}}$ value. The obtained volumes are equivalent to volumes of spheres with angstrom-sized radii.

The diffusion volume values found by Marée et al. (1996) for a-C:H are similar (slightly higher) to ours for equivalent stopping powers. In the a-C:H ion irradiation literature, a residual hydrogen content is also observed at high fluence. These residual content seems compatible with our results. The corresponding diffusion volumes were not estimated because the hydrogen densities of the different samples may not always be homogeneous, and thus $\rho_{\mathrm{f}}$ cannot be correctly determined. In previous studies, different observations were made concerning the final hydrogen content variation with the stopping power. Adel et al. (1989) report that the final hydrogen content does not depend on the ion beam used in the analysis, and that this final value is approximately 5 at. $\%$. This disagrees with the increase of the recombination volume with the stopping power observed by us and Marée et al. (1996). Baptista et al. (2004) found an opposite correlation, i.e., an increase of the final hydrogen content when the stopping cross section is raised. It should be noted, however, that some of the experiments in this study are driven by an important part of nuclear interactions, thus mixing different mechanisms at work in the interaction with ions.

\subsubsection{Dependence on the stopping power}

We found that the recombination volume $V$ increases linearly with $S_{\mathrm{e}}$. The corresponding equation for a-C:H 1 recombination volume is approximately $\left(V\right.$ in $\AA^{3}$ and $S_{\mathrm{e}}$ in $\mathrm{MeV} /\left(\mathrm{mg} / \mathrm{cm}^{2}\right)$ )

$V=2.0\left(\begin{array}{c}+1.5 \\ -1.0\end{array}\right) S_{\mathrm{e}}+50\left(\begin{array}{c}-18 \\ +20\end{array}\right)$

The best fit of $A_{\mathrm{f}} / A_{\mathrm{i}}$ corresponding to Eq. (6), called Fit 1 from now, is represented in Fig. 6 by the solid line. The dashed line represents an extreme fit, called Fit 2, for which the asymptotic $A_{\mathrm{f}} / A_{\mathrm{i}}$ are the highest for low $S_{\mathrm{e}}$ (see Eq. (6)). The fits are determined from the experimental data obtained with a-C:H 1 , but the results obtained with soot samples seem also compatible with both fits. These best and extreme fits of the residual hydrogen content at high fluence are compatible with the recombination model. Marée et al. (1996) explained that the recombination volume should be proportional to $S_{\mathrm{e}}$ if the stopping cross section is proportional to $r^{2}$ as explained above.

The minimum diffusion volumes $V$ corresponding to the maximum $A_{\mathrm{f}} / A_{\mathrm{i}}$ value, i.e. the constant in Eq. (6) of $50 \AA^{3}$ for fit 1 and $32 \AA^{3}$ for fit 2 , is also coherent with the recombination model. These minimum volume values are equivalent to the volume of a sphere with a radius of 2-3 $\AA$, i.e., the approximate distance $a$ between two adjacent hydrogen atoms ${ }^{3}$. Because the radius of the diffusion volume cannot be lower than $a$, this explains the presence of a maximum $A_{\mathrm{f}} / A_{\mathrm{i}}$ value at low $S_{\mathrm{e}}$. We can also notice that if we assume we can estimate the ion track radius $r$ from the destruction cross section $\sigma_{\mathrm{d}}$ by $\pi r^{2} \sim \sigma_{\mathrm{d}}$, the transition at $S_{\mathrm{e}} \sim 1 \mathrm{MeV} /\left(\mathrm{mg} / \mathrm{cm}^{2}\right)$, under which $A_{\mathrm{f}} / A_{\mathrm{i}}$ is roughly constant, corresponds to the $S_{\mathrm{e}}$ where the diffusion volume is equivalent to a sphere with a radius that equals the ion track radius $r$.

In the following, if nothing is indicated, the values used for $\sigma_{\mathrm{d}}$ and $A_{\mathrm{f}} / A_{\mathrm{i}}$ are those determined from the a-C:H data using the recombination model.

\footnotetext{
${ }^{3} a=\rho_{\mathrm{i}}^{-1 / 3}=2.6 \AA$ with our estimate $\rho_{\mathrm{i}}=5.6 \times 10^{22}$ at. $\mathrm{cm}^{-3}$ for $\mathrm{a}-\mathrm{C}: \mathrm{H}$.
}

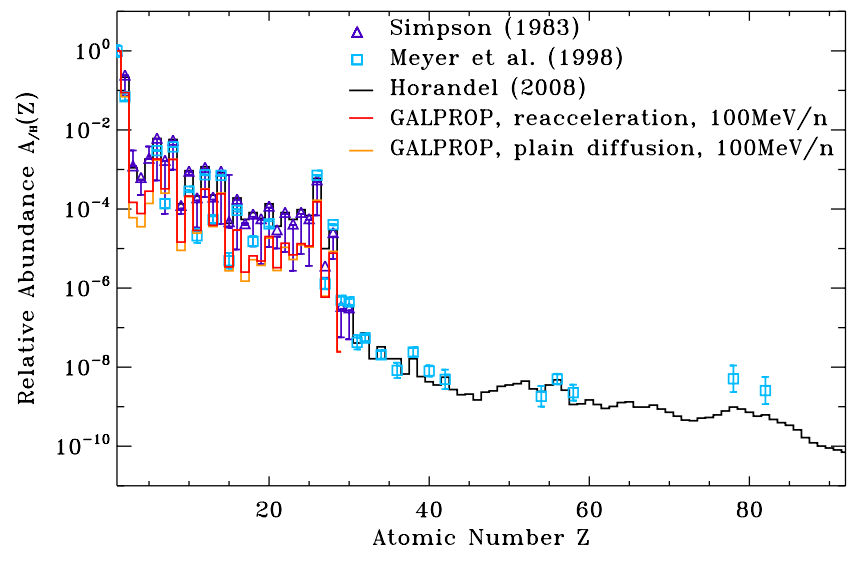

Fig. 7. Abundance of elements in cosmic rays as function of their atomic number $Z$ at energies around $0.1-1 \mathrm{GeV} /$ nucleon, normalised to hydrogen (from http://galprop.stanford.edu/, Simpson 1983; Meyer et al. 1998; Fig. 3 of Hörandel 2008, and references therein).

\section{Astrophysical implications}

Using our ion irradiation experiments, we can determine the effective aliphatic $\mathrm{C}-\mathrm{H}$ destruction cross section $\sigma_{\mathrm{d}}\left(Z, E_{\mathrm{A}}\right)$ and the asymptotic hydrogen content at high fluence $\rho_{\mathrm{f}}\left(Z, E_{\mathrm{A}}\right)$ for each ion of atomic number $Z$ and energy per nucleon $E_{\mathrm{A}}$. Below we use these results to infer the evolution of the interstellar aliphatic $\mathrm{C}-\mathrm{H}$ component exposed to cosmic rays. For this, we take the sum of the entire different cosmic rays ions and energy contributions into account.

\subsection{The interstellar cosmic ray flux}

We first need to set how many cosmic rays of each ion type and of each energy irradiate the dust components in the interstellar medium. We call $\frac{\mathrm{d} N}{\mathrm{~d} E_{\mathrm{A}}}\left(Z, E_{\mathrm{A}}\right)$ the differential flux of cosmic rays (particles $\mathrm{cm}^{-2} \mathrm{~s}^{-1} \mathrm{sr}^{-1}(\mathrm{MeV} / \text { nucleon) })^{-1}$ ) of atomic number $Z$ and energy per nucleon $E_{\mathrm{A}}$.

The $Z$ dependence of this flux is given by the cosmic ray relative abundance of each element $A_{/ \mathrm{H}}(Z)$ (normalised to hydrogen). Different distributions $A_{/ \mathrm{H}}(Z)$ found in the literature are represented in Fig. 7 (http://galprop.stanford.edu/, Simpson 1983; Meyer et al. 1998; Fig. 3 of Hörandel 2008, and references therein). Some differences exist between existing distributions in the literature, and we report their resulting effects on ionisation and destruction by cosmic rays in Table 4. We compared the results to the expected ionisation rate by cosmic rays (see Sect. 4.2) and chose to use the CR distribution at $100 \mathrm{MeV} /$ nucleon given by the GALPROP code (reacceleration model, http://galprop.stanford.edu/, Vladimirov et al. 2011). All elements lighter than copper are considered $(Z \leq 29)$.

In the following, we used the CR energy distribution given by Webber \& Yushak (1983), providing the protons differential flux $\frac{\mathrm{d} N_{\mathrm{H}}}{\mathrm{d} E_{\mathrm{A}}}\left(E_{\mathrm{A}}\right)\left(\right.$ particles $\left.\mathrm{cm}^{-2} \mathrm{~s}^{-1} \mathrm{sr}^{-1}(\mathrm{MeV} / \text { nucl) })^{-1}\right)$ as

$\frac{\mathrm{d} N_{\mathrm{H}}}{\mathrm{d} E_{\mathrm{A}}}\left(E_{\mathrm{A}}\right)=\frac{C E_{\mathrm{A}}^{0.3}}{\left(E_{\mathrm{A}}+E_{0}\right)^{3}}$,

where $E_{\mathrm{A}}$ is the cosmic ray energy per nucleon (in $\mathrm{MeV} /$ nucleon), $C=9.42 \times 10^{4}$ is a normalisation constant, and $E_{0}=400 \mathrm{MeV} /$ nucleon is a form parameter that modifies only the low-energy CR spectra. Because of the solar modulation, the distribution of low-energy CR is not directly 


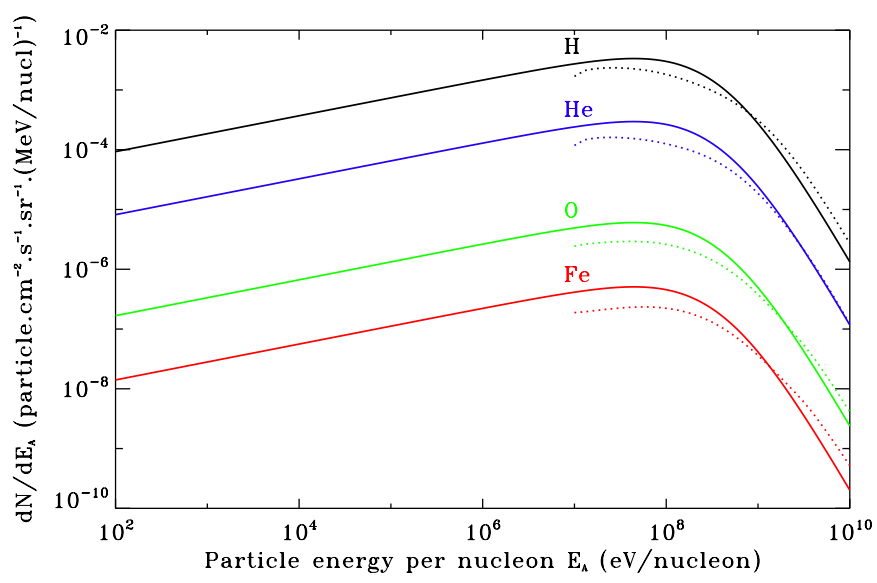

Fig. 8. Differential flux $\mathrm{d} N / \mathrm{d} E_{\mathrm{A}}$ distribution with the cosmic ray energy per nucleon $E_{\mathrm{A}}$ for some of the cosmic ray elements (hydrogen, helium, oxygen, and iron) (Webber \& Yushak 1983; Shen et al. 2004). The dotted lines are the values given by GALPROP (http://galprop. stanford. edu/webrun/, Vladimirov et al. 2011).

measurable from Earth, and is not fully determined below 10-100 MeV. Padovani et al. (2009) compared two extreme low-energy CR spectra, with CR fluxes either strongly decreasing or strongly increasing below $10 \mathrm{MeV}$. These minimum and maximum distributions are taken from Webber (1998) and Moskalenko et al. (2002), respectively, and are extrapolated below $10 \mathrm{MeV}$ by a power-law in energy. The adopted maximum distribution appears to be a very high upper limit. The distribution we adopted is between these two extremes, but more importantly, Padovani et al. (2009) have calculated that both these initial distributions obtain roughly constant CR fluxes at low energies (as the one we adopted) once the CR have traversed and interacted with interstellar matter.

The velocity spectra of different elements are quite similar to each other, hence, as in Shen et al. (2004), we assume the same normalised CR energy spectra for each element (when represented as a function of the energy per nucleon). The GALPROP CR energy distribution data (dotted lines in Fig. 8) show that this assumption is reasonable. The differential flux of cosmic rays for each element (see Fig. 8) is obtained by combining the differential energy spectra to the cosmic rays relative abundance $\frac{\mathrm{d} N}{\mathrm{~d} E_{\mathrm{A}}}\left(Z, E_{\mathrm{A}}\right)=A_{/ \mathrm{H}}(Z) \cdot \frac{\mathrm{d} N_{\mathrm{H}}}{\mathrm{d} E_{\mathrm{A}}}\left(E_{\mathrm{A}}\right)$. We consider the CR energy per nucleon $E_{\mathrm{A}}$ between $100 \mathrm{eV}$ and $10 \mathrm{GeV}$, where the CR flux is not too low and the induced destruction is efficient (out of this $E_{\mathrm{A}}$ range, the electronic stopping power is low and the destruction cross section is thus also low).

\subsection{Ionisation rate}

To check if the chosen abundance distribution is coherent, we can link the cosmic ray fluxes to an astrophysical observable. The interstellar ionisation rate by cosmic rays $R_{\mathrm{i}, \mathrm{CR}}\left(\mathrm{s}^{-1}\right)$ can be estimated with the equation

$R_{\mathrm{i}, \mathrm{CR}}=\left(1+k_{2 \mathrm{nd} e^{-}}\right) 4 \pi \sum_{Z} \int \sigma_{\mathrm{i}}\left(Z, E_{\mathrm{A}}\right) \frac{\mathrm{d} N}{\mathrm{~d} E_{\mathrm{A}}}\left(Z, E_{\mathrm{A}}\right) \mathrm{d} E_{\mathrm{A}}$,

where the cosmic ray spectra $\frac{\mathrm{d} N}{\mathrm{~d} E_{\mathrm{A}}}\left(Z, E_{\mathrm{A}}\right)$ is determined as explained in the previous section. The cross sections $\sigma_{\mathrm{i}}\left(Z, E_{\mathrm{A}}\right)\left(\mathrm{cm}^{2} /\right.$ ion $)$ for the ionisation by energetic ions are estimated from the Bethe equation reported by Spitzer \& Tomasko (1968)

$\sigma_{\mathrm{i}}\left(Z, E_{\mathrm{A}}\right)=\frac{1.23 \times 10^{-20} Z^{2}}{\beta^{2}}\left(6.20+\log _{10} \frac{\beta^{2}}{1-\beta^{2}}-0.43 \beta^{2}\right),(9)$

where $\beta \cdot c$ is the velocity of the energetic ion. $k_{2 \text { nd } e^{-}}$is the fraction of the ionisation rate due to the secondary electrons, i.e., the free electrons produced by the ionisation process and contributing then to ionise other atoms when their energy is high enough. This factor has a value around $k_{2 n d} e^{-}=0.7$ (Cravens \& Dalgarno 1978). With the adopted cosmic ray flux, we found an ionisation rate of $R_{\mathrm{i}, \mathrm{CR}}=2 \times 10^{-17} \mathrm{~s}^{-1}$.

Most of the $\mathrm{H}_{3}^{+}$observations in the diffuse interstellar medium give values of cosmic-ray ionisation rate between $2 \times$ $10^{-17} \mathrm{~s}^{-1}$ and $4 \times 10^{-16} \mathrm{~s}^{-1}$ (Webber 1998; Dalgarno 2006; Indriolo et al. 2007, 2009). However, a value of $1.2 \times 10^{-15} \mathrm{~s}^{-1}$ is inferred by McCall et al. (2003). The ionisation rate calculated from the different CR abundance distributions of Fig. 7 are all equal to a few $10^{-17} \mathrm{~s}^{-1}$. Moreover, the contribution of the CR heavy ions (other than hydrogen) in the ionisation rate is often considered to be a factor about equal to 1.8 of the contribution of hydrogen (Spitzer \& Tomasko 1968; Shen et al. 2004). This factor can be calculated as the sum $\eta=\sum A_{/ \mathrm{H}}(Z) Z^{2}$ (cf. Eqs. (8) and (9)). With the adopted GALPROP distribution, we found $\eta=1.8$, which agrees reasonably well. Table 4 shows a comparison of $\eta$ and $R_{\mathrm{i}, \mathrm{CR}}$ found with the different CR distributions $A_{/ \mathrm{H}}(Z)$.

\subsection{Destruction rate}

The destruction rate $R_{\mathrm{d}, \mathrm{CR}}\left(\mathrm{s}^{-1}\right)$ of the interstellar aliphatic $\mathrm{C}-\mathrm{H}$ component by cosmic rays can be calculated as defined by Mennella et al. (2003).

$R_{\mathrm{d}, \mathrm{CR}}=4 \pi \sum_{Z} \int \sigma_{\mathrm{d}}\left(Z, E_{\mathrm{A}}\right) \frac{\mathrm{d} N}{\mathrm{~d} E_{\mathrm{A}}}\left(Z, E_{\mathrm{A}}\right) \mathrm{d} E_{\mathrm{A}}$.

Using the SRIM code to calculate the expected stopping cross section $S_{\mathrm{e}}$ as a function of the atomic number $Z$ and of the energy per nucleon $E_{\mathrm{A}}$, and the relation established in the previous section linking $\sigma_{\mathrm{d}}\left(Z, E_{\mathrm{A}}\right)$ to $S_{\mathrm{e}}\left(Z, E_{\mathrm{A}}\right)$, we determined the aliphatic $\mathrm{C}-\mathrm{H}$ destruction cross section for each ion and energy of cosmic rays. By applying Eq. (10), we find an interstellar aliphatic C-H destruction rate by cosmic rays $R_{\mathrm{d}, \mathrm{CR}}=3.1 \times 10^{-17} \mathrm{~s}^{-1}$ (corresponding to $\alpha=1.4$ ), $R_{\mathrm{d}, \mathrm{CR}}=6.1 \times 10^{-17} \mathrm{~s}^{-1}$ (corresponding to $\alpha=1.2$ ) and $R_{\mathrm{d}, \mathrm{CR}}=1.6 \times 10^{-16} \mathrm{~s}^{-1}(\alpha=1.0)$. These values are one order of magnitude lower than the value found by Mennella et al. (2003). $R_{\mathrm{d}, \mathrm{CR}}$ found with the different CR distribution $A_{/ \mathrm{H}}(Z)$ are compared in Table 4 . This destruction rate as calculated by Mennella et al. (2003) does not consider the value of the residual $\mathrm{C}-\mathrm{H}$ aliphatic content at high fluence.

\subsection{Comprehensive description of the aliphatic $\mathrm{C}-\mathrm{H}$ evolution under interstellar cosmic ray irradiation}

We now describe in more detail the evolution of the interstellar aliphatic C-H component under cosmic ray irradiation, represented by the variable $\rho(t)$.

$$
\begin{aligned}
\rho(t+\mathrm{d} t) & =\rho(t)+\mathrm{d} \rho(t) \\
& =\rho(t)+\sum_{Z} \int \mathrm{d} \rho_{Z, E_{\mathrm{A}}}(t) \\
& =\rho(t)+\sum_{Z} \int \frac{\mathrm{d} \rho_{Z, E_{\mathrm{A}}}}{\mathrm{d} F_{\mathrm{I}}}(t) \frac{\mathrm{d} F_{\mathrm{I}}}{\mathrm{d} E_{\mathrm{A}}} \mathrm{d} E_{\mathrm{A}} .
\end{aligned}
$$


M. Godard et al.: Ion irradiation of carbonaceous interstellar analogues

Table 4. Comparison of the values of $\eta$, the ionisation and destruction rates corresponding to the different cosmic ray distribution $A_{/ \mathrm{H}}(Z)$.

\begin{tabular}{cccccc}
\hline \hline$A_{/ \mathrm{H}}(\mathrm{Z})$ distribution & $\eta$ & Ionisation rate $\left(\mathrm{s}^{-1}\right)$ & \multicolumn{3}{c}{ Destruction rate $\left(\mathrm{s}^{-1}\right)$} \\
& & & $\alpha=1.0$ & $\alpha=1.2$ & $\alpha=1.4$ \\
\hline GALPROP reacceleration model & 1.84 & $2.3 \times 10^{-17}$ & $1.6 \times 10^{-16}$ & $6.1 \times 10^{-17}$ & $3.1 \times 10^{-17}$ \\
GALPROP plain diffusion model & 1.76 & $2.2 \times 10^{-17}$ & $1.5 \times 10^{-16}$ & $5.9 \times 10^{-17}$ & $3.0 \times 10^{-17}$ \\
Meyer et al. (1998) & 2.47 & $3.1 \times 10^{-17}$ & $2.1 \times 10^{-16}$ & $1.0 \times 10^{-16}$ & $6.5 \times 10^{-17}$ \\
Simpson (1983) & 3.93 & $4.9 \times 10^{-17}$ & $3.3 \times 10^{-16}$ & $1.6 \times 10^{-16}$ & $9.9 \times 10^{-17}$ \\
Hörandel (2008) & 3.88 & $4.9 \times 10^{-17}$ & $3.2 \times 10^{-16}$ & $1.6 \times 10^{-16}$ & $1.0 \times 10^{-16}$ \\
\hline
\end{tabular}

Table 5. Percentage of destruction $\%_{\mathrm{d}}$ at a typical cloud time scale $t=$ $3 \times 10^{7} \mathrm{yrs}$ for the different fits adopted concerning $\sigma_{\mathrm{d}}$ and $A_{\mathrm{f}} / A_{\mathrm{i}}$.

\begin{tabular}{cccc}
\hline \hline Fits adopted & $A_{\mathrm{f}} / A_{\mathrm{i}}=0$ & $A_{\mathrm{f}} / A_{\mathrm{i}}:$ Fit 1 & $A_{\mathrm{f}} / A_{\mathrm{i}}:$ Fit 2 \\
\hline$\sigma_{\mathrm{d}}: \alpha=1.0$ & $14 \%$ & $21 \%$ & $13 \%$ \\
$\sigma_{\mathrm{d}}: \alpha=1.2$ & $6 \%$ & $10 \%$ & $7 \%$ \\
$\sigma_{\mathrm{d}}: \alpha=1.4$ & $3 \%$ & $6 \%$ & $5 \%$ \\
\hline
\end{tabular}

The units of $\frac{\mathrm{d} F_{\mathrm{I}}}{\mathrm{d} E_{\mathrm{A}}}$ are ions $\mathrm{cm}^{-2}(\mathrm{MeV} / \mathrm{nucl})^{-1}$ and this variable can be expressed as a function of the cosmic ray differential flux:

$\frac{\mathrm{d} F_{\mathrm{I}}}{\mathrm{d} E_{\mathrm{A}}}=\frac{\mathrm{d} N}{\mathrm{~d} E_{\mathrm{A}}} \cdot \mathrm{d} t \cdot 4 \pi$.

The equation describing the evolution of the aliphatic $\mathrm{C}-\mathrm{H}$ concentration in interstellar materials becomes

$\rho(t+\mathrm{d} t)=\rho(t)+\mathrm{d} t \frac{\mathrm{d} \rho}{\mathrm{d} t}(t)$

with $\frac{\mathrm{d} \rho}{\mathrm{d} t}(t)=4 \pi \sum_{Z} \int \frac{\mathrm{d} \rho_{Z, E_{\mathrm{A}}}}{\mathrm{d} F_{\mathrm{I}}}(t) \frac{\mathrm{d} N}{\mathrm{~d} E_{\mathrm{A}}}\left(Z, E_{\mathrm{A}}\right) \mathrm{d} E_{\mathrm{A}}$.

The value $\frac{\mathrm{d} \rho}{\mathrm{d} t}(t)$ represents the destruction of the interstellar $\mathrm{C}-\mathrm{H}$ aliphatic component at instant $t$ by the whole distribution of cosmic ray ions and energies. For the recombination model, we can write

$\frac{\mathrm{d} \rho_{Z, E_{\mathrm{A}}}}{\mathrm{d} F_{\mathrm{I}}}(t)= \begin{cases}0 & \text { if } \rho(t) \leq \rho_{\mathrm{f}}\left(Z, E_{\mathrm{A}}\right) \\ -\sigma_{\mathrm{d}}\left(Z, E_{\mathrm{A}}\right) \cdot \rho(t) \cdot\left(\frac{\rho(t)}{\rho_{\mathrm{f}}\left(Z, E_{\mathrm{A}}\right)}-1\right) & \text { if } \rho(t)>\rho_{\mathrm{f}}\left(Z, E_{\mathrm{A}}\right) .\end{cases}$

When $\rho(t)$ is beyond the asymptotic value $\rho_{\mathrm{f}}\left(Z, E_{\mathrm{A}}\right)$, the cosmic rays of atomic number $Z$ and of energy per nucleon $E_{\mathrm{A}}$ do not participate anymore in the destruction. As a result, $\frac{\mathrm{d} \rho_{Z, E_{\mathrm{A}}}}{\mathrm{d} F_{\mathrm{I}}}(t)=0$ for $\rho(t) \leq \rho_{\mathrm{f}}\left(Z, E_{\mathrm{A}}\right)$. The $\sigma_{\mathrm{d}}\left(Z, E_{\mathrm{A}}\right)$ and $\rho_{\mathrm{f}}\left(Z, E_{\mathrm{A}}\right)$ values are calculated for each ion and energy from the behaviour of irradiated a-C:H samples with the stopping cross section $S_{\mathrm{e}}$ (Figs. 5 and 6 , respectively). The temporal evolution of the $\mathrm{C}-\mathrm{H}$ aliphatic component of the interstellar matter under cosmic ray is represented in Fig. 9. Note that the destruction rate $R_{\mathrm{d}, \mathrm{CR}}$ defined above corresponds to the value of $-\frac{1}{\rho} \frac{\mathrm{d} \rho}{\mathrm{d} t}$ if $\rho$ follows an exponential decrease with $\rho_{\mathrm{f}}=0$. This exponential decrease $\rho / \rho_{\mathrm{i}}=$ $\exp \left(-R_{\mathrm{d}, \mathrm{CR}} \cdot t\right)$ (only the values of $\sigma_{\mathrm{d}}\left(Z, E_{\mathrm{A}}\right)$ are considered in the previous equations, and $\left.\rho_{\mathrm{f}}\left(Z, E_{\mathrm{A}}\right)=0\right)$ is also reported in Fig. 9 (black line) for sake of comparison. The relative destruction of the $\mathrm{C}-\mathrm{H}$ aliphatic component $\%_{\mathrm{d}}$ corresponding to a typical cloud time scale $t=3 \times 10^{7}$ years (McKee 1989; Draine 1990; Jones et al. 1994) are given in Table 5 for the different adopted fits.

\subsection{Heavy ions contribution}

In the destruction rate calculation (i.e., exponential decrease not taking into account the residual hydrogen content), the

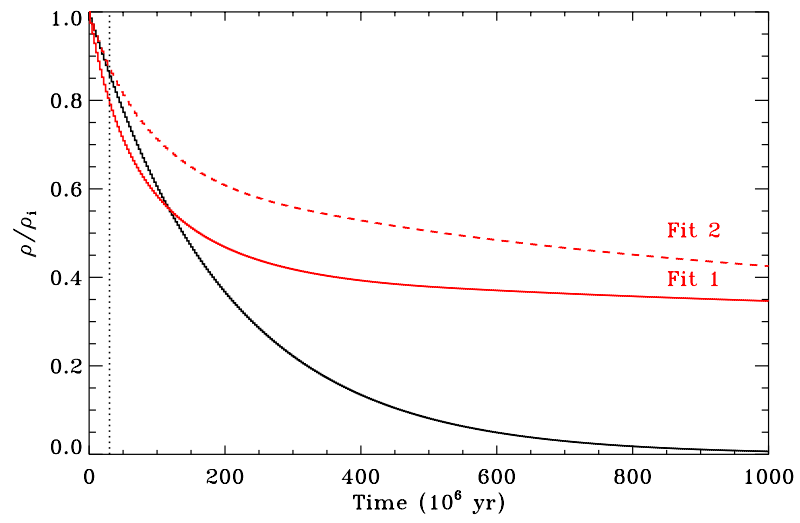

Fig. 9. Evolution of the $\mathrm{C}-\mathrm{H}$ aliphatic component of the interstellar matter under cosmic ray irradiation. The values of $\sigma_{\mathrm{d}}$ were determined from a-C:H 1 data with $\alpha=1.0$ (see Fig. 5). The black curve corresponds to an exponential decrease without residual hydrogen content taking into account $\left(\rho / \rho_{\mathrm{i}}=\exp \left(-R_{\mathrm{d}, \mathrm{CR}} \cdot t\right)\right)$. The red solid (fit 1) and dashed (fit 2) curves are obtained when the asymptotic values $\rho_{\mathrm{f}} / \rho_{\mathrm{i}} \neq 0$ are considered. Values of $\rho_{\mathrm{f}} / \rho_{\mathrm{i}}$ are determined from a-C:H 1 data and two different fits (see Fig. 6 and text for details). The dotted vertical line corresponds to a typical cloud time scale $t=3 \times 10^{7} \mathrm{yr}$. (McKee 1989; Draine 1990; Jones et al. 1994).

cosmic ray heavy ions contribution to ionisation and destruction are constant in time. These contributions are summarised in Table 6.

Taking into account the residual hydrogen content after long irradiation, the destruction caused by the contribution of ions heavier than hydrogen and helium increases with time (see Fig. 10). The contribution of abundant light CR elements becomes insignificant when the corresponding $A_{\mathrm{f}} / A_{\mathrm{i}}$ is reached, and then, only heavier elements pursue the destruction. In Fig. 10 we can see that for $\alpha=1.0$, this transition where heavy elements begin to dominate the destruction occurs after irradiation of few hundreds of million years. This corresponds to a time greater than or of the same order as an interstellar cloud lifetime. When $\alpha=1.4$, heavy elements contribution is dominant from the beginning of the CR exposure. This is the case when $\alpha \geq 1.3$ for the fit 1 , and when $\alpha \geq 1.2$ for the fit 2 .

In every case, the contribution of heavy elements, and particularly of iron ions, is important. Although the Fe ions are less abundant by four orders of magnitude than hydrogen in cosmic rays, they represent a contribution to the aliphatic $\mathrm{C}-\mathrm{H}$ destruction by cosmic rays between $5 \%$ and $40 \%$ (with $t \leq$ $10^{8}$ years).

\subsection{Evolution of the $3.4 \mu \mathrm{m}$ feature between diffuse and dense phases of interstellar medium}

In this paragraph we discuss the contribution of cosmic rays in the destruction of the interstellar aliphatic $\mathrm{C}-\mathrm{H}$ component. This 
Table 6. Contribution of each major $\mathrm{CR}$ element in the abundance, ionisation, and destruction when $A_{\mathrm{f}} / A_{\mathrm{i}}=0$ is considered.

\begin{tabular}{cccccc}
\hline \hline Element & $\begin{array}{c}\text { Abundance } \\
(\%)\end{array}$ & $\begin{array}{c}\text { Ionisation } \\
(\%)\end{array}$ & \multicolumn{3}{c}{$\begin{array}{c}\text { Destruction, for } A_{\mathrm{f}} / A_{\mathrm{i}}=0 \\
(\%)\end{array}$} \\
& & & $\alpha=1.0$ & $\alpha=1.2$ & $\alpha=1.4$ \\
\hline $\mathrm{H}$ & 91.4 & 54.3 & 55.7 & 38.7 & 22.7 \\
$\mathrm{He}$ & 8.1 & 19.1 & 19.5 & 17.8 & 13.6 \\
$\mathrm{O}$ & 0.2 & 6.3 & 6.1 & 9.3 & 11.4 \\
$\mathrm{Fe}$ & 0.01 & 5.6 & 4.8 & 10.8 & 19.3 \\
\hline
\end{tabular}

Notes. The cosmic ray elemental abundances used are those given by GALPROP, at $100 \mathrm{MeV}$ per nucleon, using the "reacceleration model". The contribution of different $\mathrm{CR}$ elements in the destruction, when $A_{\mathrm{f}} / A_{\mathrm{i}} \neq 0$ is considered, can be seen in Fig. 10 .
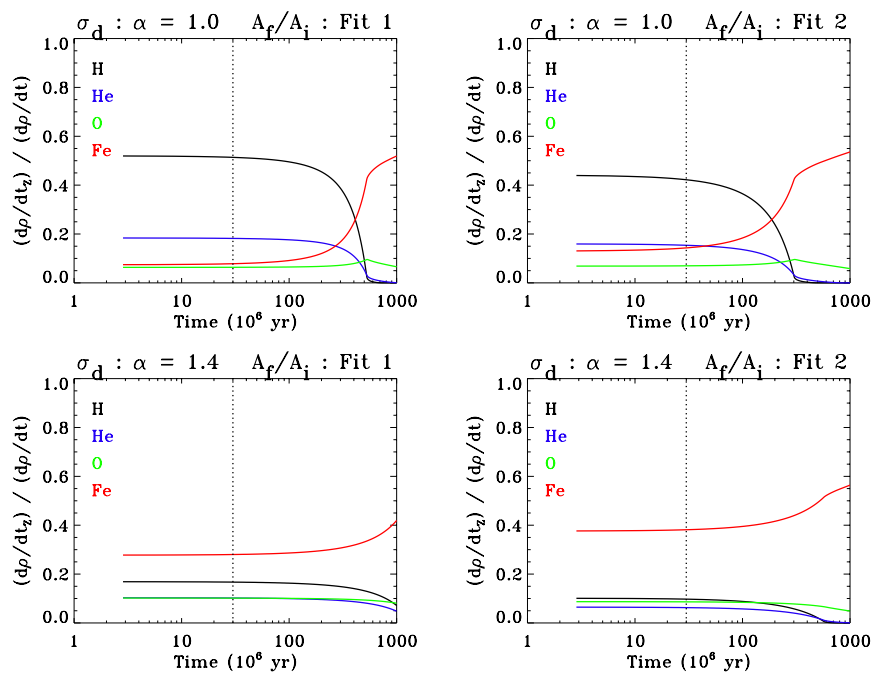

Fig. 10. Variation of $\frac{\mathrm{d} \rho}{\mathrm{d} t}(Z) / \frac{\mathrm{d} \rho}{\mathrm{d} t}$, the part of the destruction caused by element of atomic number $Z$ at instant $t$, as a function of the time for the few major cosmic ray elements. The dotted vertical lines correspond to a typical cloud time scale $t=3 \times 10^{7}$ yr. (McKee 1989; Draine 1990; Jones et al. 1994).

will be compared to the interstellar destruction by UV photons, and to the formation by $\mathrm{H}$ atoms exposure. These processes are confronted in the different environments of the ISM. The examination of the diffuse and dense interstellar regions will show the need to consider the intermediate regions, too. This discussion is summarised in Table 7.

As shown in Fig. 9, the characteristic destruction time $t_{\mathrm{d}, \mathrm{CR}}$ of the interstellar $3.4 \mu \mathrm{m}$ absorption feature by cosmic rays is of few $10^{8}$ years, a time greater than the typical lifetime of interstellar clouds $t_{\text {cloud }}=3 \times 10^{7} \mathrm{yr}$. Moreover, by considering in an astrophysical model the asymptotic values at infinite fluence $A_{\mathrm{f}} \neq 0$, we found that, even at a longer time, the destruction by cosmic rays is not total (after $1 \times 10^{9} \mathrm{yr}$, the destruction did not go further than $65 \%$ ). These results are valid for both the diffuse and dense interstellar medium because the CR flux is the same in both regions. This clearly confirms that cosmic ray irradiation cannot be the only process responsible for the $3.4 \mu \mathrm{m}$ absorption feature disappearance in the dense interstellar medium.

Indeed, when they are present, the UV photons dominate the destruction. The destruction of $\mathrm{C}-\mathrm{H}$ bonds by UV irradiation has been studied by Muñoz Caro et al. (2001) in hydrocarbon molecules and by Mennella et al. (2001) in hydrocarbon grains. They found a destruction cross section $\sigma_{\mathrm{d}, \mathrm{UV}}=1.0 \times$ $10^{-19} \mathrm{~cm}^{2} /$ photon. They also found that this dehydrogenation process is still efficient with a thin ice layer, without apparent modification of $\sigma_{\mathrm{d}, \mathrm{UV}}$.

\subsubsection{Diffuse interstellar medium}

In diffuse clouds, an interstellar radiation flux of about $8 \times$ $10^{7}$ photons $\mathrm{cm}^{-2} \mathrm{~s}^{-1}$ (Mathis et al. 1983), combined with $\sigma_{\mathrm{d}, \mathrm{UV}}$ found by Mennella et al. (2001), gives a characteristic destruction time by exposure to UV radiation $t_{\mathrm{d}, \mathrm{UV}}^{\text {diffuse }}=4 \times 10^{3}$ years, a time much shorter than for the destruction by cosmic rays. The destruction of the interstellar $3.4 \mu \mathrm{m}$ feature in diffuse medium is thus most probably caused by the UV field in these regions rather than by $\mathrm{CR}$ interactions. The ubiquitous presence of the aliphatic $3.4 \mu \mathrm{m}$ feature in the diffuse interstellar medium requires an efficient formation mechanism in these regions to balance the destruction by UV and cosmic rays, possibly by exposure to atomic hydrogen (Mennella et al. 1999, 2002; Mennella 2006). Mennella (2006) found a cross section of C-H bond formation by exposure to $\mathrm{H}$ atoms $\sigma_{\mathrm{f}, \mathrm{H}}=1.7 \times 10^{-18} \mathrm{~cm}^{2} / \mathrm{H}$ atom for a temperature of $100 \mathrm{~K}$ of $\mathrm{H}$ atoms in diffuse clouds (this cross section decreases when the temperature drops). A flux of $8 \times 10^{6} \mathrm{H}$ atoms $\mathrm{cm}^{-2} \mathrm{~s}^{-1}$ in diffuse clouds (Sorrell 1990) corresponds to a characteristic formation time in diffuse clouds $t_{\mathrm{f}, \mathrm{H}}^{\mathrm{diffuse}}=2 \times 10^{3} \mathrm{yr}$. Thus, in diffuse clouds, the evolution of the interstellar $3.4 \mu \mathrm{m}$ absorption feature is governed by its formation due to exposure to $\mathrm{H}$ atoms, and its destruction mainly due to UV irradiation. The resulting balance between formation and destruction is in favour of the formation in these diffuse regions.

\subsubsection{Dense interstellar medium}

In dense molecular clouds, the $3.4 \mu \mathrm{m}$ feature with a profile associated with the methyl and methylene substructures, which is characteristic of diffuse ISM hydrocarbons, is not observed. This $3.4 \mu \mathrm{m}$ band with substructures must not be confused with the featureless broad band at $3.47 \mu \mathrm{m}$ that is seen in dense clouds, and whose the optical depth is correlated with the water ice one. Observations with high signal-to-noise ratio show that the upper limit on the $3.4 \mu \mathrm{m}$ optical depth in dense clouds is low. It suggests that the carriers of this band are absent from these regions, or at most present in very small quantities.

In dense clouds, the $3.4 \mu \mathrm{m}$ feature formation process is impeded by several factors: (1) most of the hydrogen is in molecular form, the flux of $\mathrm{H}$ atoms is reduced by two orders of magnitude compared to diffuse ISM. (2) Dust grains in dense regions are expected to be covered by an ice layer that prevents the hydrogenation of the material by $\mathrm{H}$ atoms. (3) The formation cross section for a temperature of $10 \mathrm{~K}$ of $\mathrm{H}$ atoms in dense clouds is expected to be three orders of magnitude lower than for the $\mathrm{H}$ atom temperature in diffuse clouds (Mennella 2006). Therefore, the methyl and methylene aliphatic $\mathrm{C}-\mathrm{H}$ formation process probably remains ineffective in dense regions, while the destruction by cosmic rays still proceeds (the presence of an ice layer does not seem to affect the destruction cross section by $\mathrm{CR}$ as shown by Mennella et al. (2003)).

Recently, another hypothesis concerning the exposure to $\mathrm{H}$ atoms in dense clouds has been proposed in Mennella (2008) and Mennella (2010). He exposed carbon particles covered with a water ice layer to $\mathrm{H}$ atoms. These experiments, under simulated dense ISM conditions, result in no observed formation of aliphatic $\mathrm{C}-\mathrm{H}$ bonds in the $\mathrm{CH}_{2}$ and $\mathrm{CH}_{3}$ functional groups (i.e., those responsible for the $3.4 \mu \mathrm{m}$ absorption feature observed in diffuse ISM), but a large absorption band around 
Table 7. Synthetic table giving the characteristic times of destruction and formation processes of the aliphatic hydrogenated carbon component in the different phases of the interstellar medium.

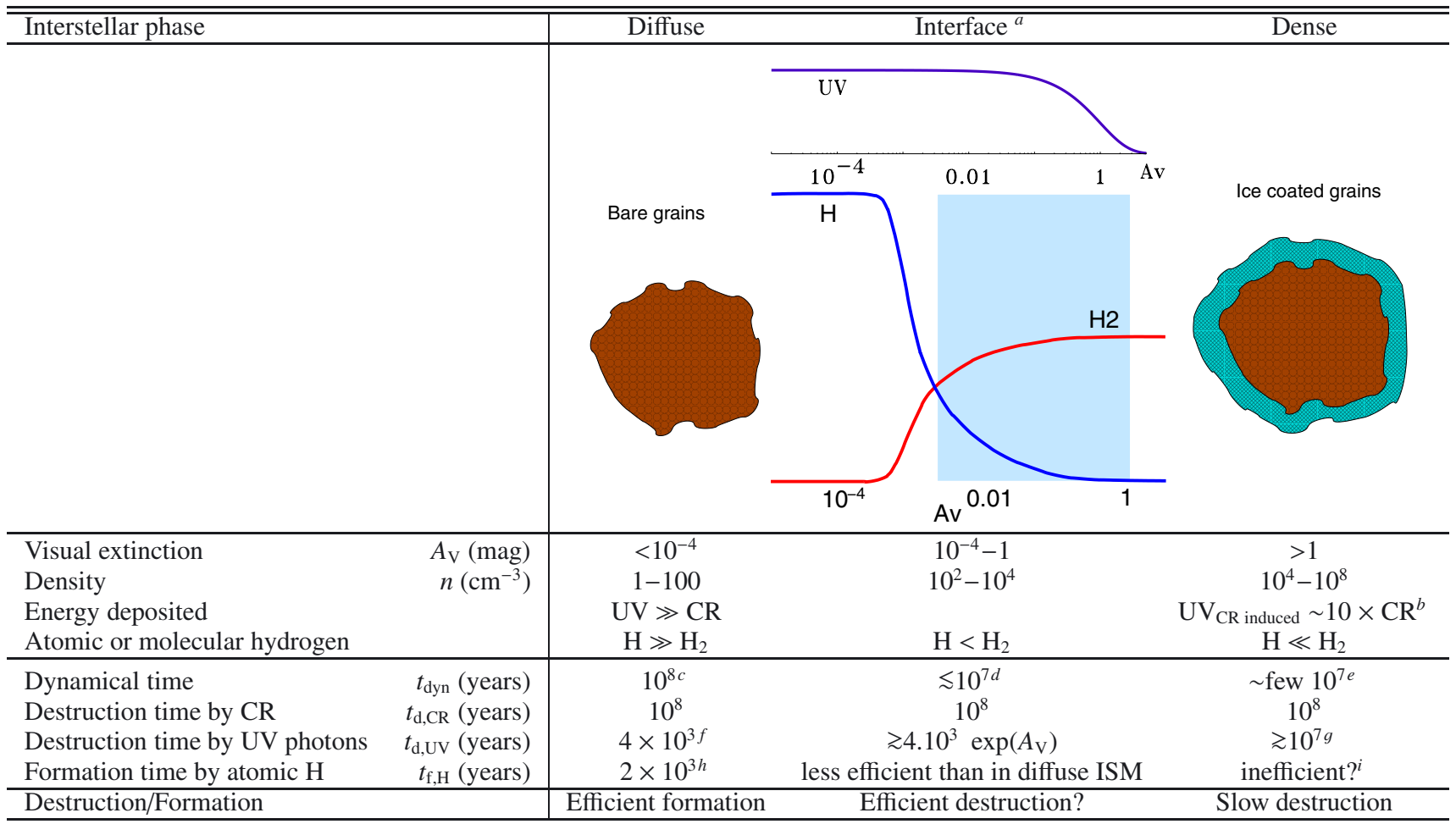

Notes. The destruction and formation characteristic times can be compared to the dynamical time, i.e., the approximate time spent in each of these phases. ${ }^{(a)}$ The figure is adapted from Le Petit et al. (2006). The blue zone emphasises the transition zone between atomic and molecular hydrogen. ${ }^{(b)}$ The local UV field induced by cosmic rays deposits more energy than cosmic rays themselves (Shen et al. 2004). However, because the penetration depth of photons is smaller, the ice mantles are more UV processed, whereas the internal part of grains is dominated by cosmic ray energy deposition. ${ }^{(c)}$ E.g., Jones et al. (1994). ${ }^{(d)}$ E.g., Goldsmith et al. (2007); Glover \& Mac Low (2007). ${ }^{(e)}$ McKee (1989); Draine (1990); Jones et al. (1994); Tassis \& Mouschovias (2004); Mouschovias et al. (2006); Elmegreen (2007). ${ }^{(f)}$ Mennella et al. $(2001)\left(\sigma_{\mathrm{d}, \mathrm{UV}}=1.0 \times\right.$ $10^{-19} \mathrm{~cm}^{2} /$ photon), Mathis et al. (1983) (interstellar radiation flux of about $8 \times 10^{7}$ photons $\left.\mathrm{cm}^{-2} \mathrm{~s}^{-1}\right)$. ${ }^{(g)}$ Mennella et al. $(2001)\left(\sigma_{\mathrm{d}, \mathrm{Uv}}=\right.$ $1.0 \times 10^{-19} \mathrm{~cm}^{2} /$ photon), Prasad \& Tarafdar (1983) (flux of internal UV field due to CR of $10^{3}-10^{4}$ photons $\mathrm{cm}^{-2} \mathrm{~s}^{-1}$ ). This results in a characteristic destruction time by an internal UV field of $10^{7}-10^{8}$ yr. Once the ice mantles appear on the dust grains $\left(A_{\mathrm{V}} \gtrsim 3\right.$, (Whittet et al. 1988; Smith et al. 1993; Murakawa et al. 2000)), the refractory material is partly protected from the UV via the photochemical interaction in these mantles. (h) Mennella (2006) $\left(\sigma_{\mathrm{f}, \mathrm{H}}=1.7 \times 10^{-18} \mathrm{~cm}^{2} / \mathrm{H}\right.$ atom), Sorrell (1990) (flux of $8 \times 10^{6} \mathrm{H}$ atoms cm $\mathrm{s} \mathrm{s}^{-1}$ in diffuse clouds).

${ }^{(i)}$ See the first two paragraphs of Sect. 4.6.2 for details.

$3.47 \mu \mathrm{m}$ appears in the IR spectrum. He assigned this band to the $\mathrm{C}-\mathrm{H}$ stretching vibration of tertiary $\mathrm{sp}^{3}$ carbon atoms and proposed that the interstellar aliphatic $3.4 \mu \mathrm{m}$ band observed in diffuse regions becomes the $3.47 \mu \mathrm{m}$ in dense clouds after interstellar processing.

Although the $3.4 \mu \mathrm{m}$ feature destruction prevails in dense interstellar medium, this work shows that cosmic rays alone cannot account for its apparent disappearance within the cloud lifetime. The galactic UV field cannot penetrate the dense clouds and thus cannot account for an additional destruction. Interestingly, the interaction of galactic cosmic rays with the interstellar medium creates an internal UV field owing to CR-induced $\mathrm{H}_{2}$ fluorescence (Prasad \& Tarafdar 1983). The corresponding flux is estimated to be of about $10^{3}-10^{4}$ photons $\mathrm{cm}^{-2} \mathrm{~s}^{-1}$. The related destruction time is $\sim 10^{7}-10^{8}$ years. Above a certain $A_{\mathrm{V}}$, when ices appear, a majority of these UV photons is probably absorbed by the ice layers and does not affect the aliphatic $\mathrm{C}-\mathrm{H}$ component. In dense clouds, the destruction time by internal UV field is thus longer than $\sim 10^{7}-10^{8}$ years, except when the ice does not cover the dust grains yet, i.e. for $A_{\mathrm{V}} \lesssim 3$ (Whittet et al. 1988; Smith et al. 1993; Murakawa et al. 2000). In this case, the effect of UV induced by CR can be 10 times greater than that of CR themselves. This internal UV field is thus a non negligible indirect destruction process induced by cosmic rays, but still does not seem to be enough to account for the entire (or almost entire) $3.4 \mu \mathrm{m}$ destruction.

\subsubsection{Interface between diffuse and dense regions}

It seems that the disappearance of the $3.4 \mu \mathrm{m}$ absorption band does not occur in either dense molecular clouds or in the diffuse ISM, but rather in intermediate regions, such as translucent clouds and photon dominated regions. In these interstellar phases, the recombination of $\mathrm{H}$ atoms into molecular hydrogen occurs and the atomic hydrogen quantity begins to decrease for visual extinction $A_{\mathrm{V}} \sim 10^{-4}-10^{-3}$ (Le Petit et al. 2006). The lower abundance of $\mathrm{H}$ atoms makes the "rehydrogenation" become less efficient in these regions, with a characteristic formation time that is probably much longer. The external UV field decreases as $\exp \left(-k A_{\mathrm{V}}\right)$. As shown in Table 7 , between $A_{\mathrm{V}} \sim$ $10^{-3}$ and $A_{\mathrm{V}}$ of a few magnitude units, the atomic hydrogen quantity is lower than in diffuse ISM, and the UV flux is still 
intense to dehydrogenate the $3.4 \mu \mathrm{m}$ feature carriers. In these regions, the destruction of this component should be efficient enough to make the $3.4 \mu \mathrm{m}$ feature disappear in dense regions. Unfortunately, in these regions at the interface between diffuse and dense ISM, observations would not easily provide additional constraints on this scenario because an eventual $3.4 \mu \mathrm{m}$ absorption feature would be masked by other strong emission features. Mennella et al. $(2001,2003)$ have proposed the material circulation in the dense cloud (only $1 \%$ of the cloud lifetime in the cloud edges is necessary to process the dust by external UV field) or the penetration of the external UV field in filamentary structures as alternative possible explanations.

\section{Conclusion}

We have performed experiments of irradiation by energetic ions of carbonaceous interstellar analogues (a-C:H and soot). The progressive dehydrogenation of the materials induced by the irradiations was measured through the monitoring of the aliphatic $\mathrm{C}-\mathrm{H}$ vibration bands in the near- and mid-infrared. The topic has been previously investigated by Mennella et al. (2003) using low-energy ion irradiation (30 keV He ions) coupled to an extrapolation. In this study, we went beyond by addressing in particular the following points: (i) different elements and energies were used, allowing us to explore a wide range of electronic stopping cross sections, comparable to those of cosmic rays. (ii) The recombination model, which explains the observed dehydrogenation through the molecular recombination of two liberated hydrogen atoms in the bulk of the irradiated material, was used to extract the characteristic $\mathrm{C}-\mathrm{H}$ destruction parameters (destruction cross section and residual hydrogen content at high fluence). (iii) The behaviour of these parameters with the electronic stopping cross section was deduced from our experiments. (iv) In particular, the observed residual hydrogen content at infinite irradiation dose, in agreement with the recombination model, were taken into account in our analysis, and we found that the corresponding recombination volumes are related to the electronic stopping cross section by a linear expression. These results were used to infer the effect of cosmic rays on the interstellar aliphatic C-H component. (v) Although a-C:H and soot samples have a different structure and hydrogen content, no significant difference in the destruction parameters was observed, suggesting that a slightly different material than our a-C:H samples as carrier for the $3.4 \mu \mathrm{m}$ band would not modify these results.

We showed that the destruction of aliphatic $\mathrm{C}-\mathrm{H}$ bonds by cosmic rays occurs in characteristic times of few $10^{8}$ years and is not complete even at longer time scales. The contribution of heavy elements (of the iron element in particular) to this destruction is important. We compared these results to the processing by UV photons and $\mathrm{H}$ atoms in the different interstellar phases. As shown by Mennella et al. (2003), in the diffuse ISM the destruction by cosmic rays is negligible compared to the destruction by UV radiation. The hydrogenation by $\mathrm{H}$ atoms exceeds the dehydrogenation (mainly by UV photons) in these regions, which explains the $3.4 \mu \mathrm{m}$ band observations. In dense clouds, the formation by $\mathrm{H}$ atoms exposure is ineffective, the galactic UV field cannot penetrate, and only destruction by cosmic rays (directly and indirectly through the created internal UV field) can process the dust grains. However, the diffuse ISM $3.4 \mu \mathrm{m}$ band is not observed in dense regions and the cosmic rays only cannot account for this disappearance. We conclude that the destruction (or modification) of the interstellar aliphatic $\mathrm{C}-\mathrm{H}$ component most probably occurs in intermediate regions, where UV and cosmic ray destruction dominate the formation by hydrogen atoms.

The irradiation of interstellar dust by cosmic rays is not the most efficient process to dehydrogenate, but cosmic rays are almost never shielded, and this processing is thus present in all interstellar regions. To fully understand the evolution of interstellar dust under this energetic processing, some work still needs to be done. In the experiments presented here, we took care to irradiate our samples with low fluxes to avoid heating the materials. Even if the temperature effect should not be major, it would be interesting to perform these experiments in conditions closer to those of the interstellar medium, i.e., at low temperature (the hydrogen mobility decrease could influence the value of the destruction parameters). The destruction effect of secondary electrons (created by the cosmic rays) was not taken into account in this work. These low-energy electrons $(\sim 30 \mathrm{eV})$ have a short projected range. In dense regions, they are stopped in the ice mantles, and only alter the surface of dust grains in diffuse regions. Even if only a small fraction of the dust in diffuse medium is affected, it would be interesting to explore the effect of these secondary electrons. The recombination model we used to describe the dehydrogenation process is valid when the interaction of the ion can be considered to be continuous along the track in the material. This could not be the case for ions with low stopping power. More irradiation experiments with high fluence of these ions would allow to check if a different regime occurs at low $S_{\mathrm{e}}$. Moreover, we only discuss the destruction of the aliphatic C-H bonds, but we saw the variation of other IR bands under ion irradiation. Another step in the study will explore these variations, which should give us information about the structural modifications induced by ion irradiation.

Acknowledgements. We would like to thank Dominique Deboffle, Philippe Duret, Thierry Redon and Raymond Vasquez for their help with the experimental developments, Vincent Guerrini for making dedicated vacuum vessels, and Pierre de Marcellus for allowing us to borrow the IR spectrometer. Finally, we aknowledge G. Muñoz Caro for helpful comments on this paper. This work was in part supported by the ANR COSMISME project, grant ANR-2010-BLAN0502 of the French Agence Nationale de la Recherche, by the French INSUCNRS national program "Physique et Chimie du Milieu Interstellaire" (PCMI), and by the Paris Sud University "Programme Pluri-Formation" (PPF). This research has made use of NASA's Astrophysics Data System.

\section{Appendix A: The exponential fit}

Alternatively, the hydrogen concentration decrease seen through IR absorption bands or other analysis methods can also be fitted assuming a first-order kinetic process (Mennella et al. 2003; Pawlak et al. 1997), with an exponential function and an added constant representing the aliphatic feature residual intensity at high ion fluences. This fit equation is

$\rho=\rho_{\mathrm{f}}+\left(\rho_{\mathrm{i}}-\rho_{\mathrm{f}}\right) \exp \left(-\sigma_{\mathrm{d}} F_{\mathrm{I}}\right)$.

The corresponding rate equation for the hydrogen density as a function of the ion fluence is

$\frac{\mathrm{d} \rho}{\mathrm{d} F_{\mathrm{I}}}=-\sigma_{\mathrm{d}} \rho\left(1-\frac{\rho_{\mathrm{f}}}{\rho}\right)$.

This equation can be used with the IR feature integrated optical depth $A$ instead of the hydrogen concentration $\rho$ of the material. It is a simply phenomenological function that does not result from a model. 
Table C.1. Results of ion irradiation experiments obtained with the exponential fits.

\begin{tabular}{ccccccc}
\hline \hline Sample & Ion & $\begin{array}{c}E \\
(\mathrm{MeV})\end{array}$ & $\begin{array}{c}S_{\mathrm{e}} \\
\left(\mathrm{MeV} /\left(\mathrm{mg} / \mathrm{cm}^{2}\right)\right)\end{array}$ & $\begin{array}{c}\sigma_{\mathrm{d}} \\
\left(\mathrm{cm}^{2}\right)\end{array}$ & $\begin{array}{c}A_{\mathrm{f}} / A_{\mathrm{i}} \\
1 / \rho_{\mathrm{f}} \\
\left(\AA^{3}\right)\end{array}$ \\
\hline a-C:H 1 & $\mathrm{H}^{+}$ & 10 & $4.580 \times 10^{-2}$ & $(0.05) 1.9(3.5) \times 10^{-16}$ & - & - \\
a-C:H 1 & $\mathrm{H}^{+}$ & 10 & $4.580 \times 10^{-2}$ & $(0.7) 3.2(7.0) \times 10^{-17}$ & $(0.00) 0.72(0.83)$ & $>21$ \\
a-C:H 1 & $\mathrm{He}^{2+}$ & 20 & $3.207 \times 10^{-1}$ & $(0.4) 3.8(5.3) \times 10^{-15}$ & - & - \\
a-C:H 1 & $\mathrm{H}^{+}$ & 0.2 & $7.092 \times 10^{-1}$ & $(0.8) 2.6(5.5) \times 10^{-16}$ & $(0.26) 0.56(0.64)$ & $(28) 32(69)$ \\
a-C:H 1 & $\mathrm{C}^{6+}$ & 91 & 2.07 & $(3.7) 9.4(17) \times 10^{-16}$ & $(0.29) 0.45(0.50)$ & $(35) 40(61)$ \\
a-C:H 1 & $\mathrm{C}^{5+}$ & 50 & 3.274 & $(1.5) 3.4(6.1) \times 10^{-15}$ & $(0.42) 0.52(0.56)$ & $(32) 34(42)$ \\
a-C:H 1 & $\mathrm{Si}^{7+}$ & 85 & 15.91 & $(1.6) 3.6(5.7) \times 10^{-14}$ & $(0.21) 0.30(0.31)$ & $(58) 59(82)$ \\
a-C:H 1 & $\mathrm{Ni}^{9+}$ & 100 & 46.98 & $(1.2) 2.1(2.9) \times 10^{-13}$ & $<0.45$ & $>40$ \\
a-C:H 1 & $\mathrm{I}^{12+}$ & 160 & 89.33 & $(2.6) 4.6(7.2) \times 10^{-13}$ & $(0.24) 0.31(0.36)$ & $(50) 57(72)$ \\
a-C:H 2 & $\mathrm{He}^{2+}$ & 20 & $3.207 \times 10^{-1}$ & $<1.0 \times 10^{-15}$ & - & - \\
a-C:H 2 & $\mathrm{C}^{6+}$ & 91 & 2.07 & $(1.0) 1.9(3.1) \times 10^{-15}$ & $(0.56) 0.63(0.68)$ & $(26) 28(32)$ \\
a-C:H 2 & $\mathrm{I}^{12+}$ & 160 & 89.33 & $(1.3) 6.1(18) \times 10^{-13}$ & $(0.00) 0.54(0.71)$ & $>25$ \\
Soot & $\mathrm{H}^{+}$ & 10 & $4.109 \times 10^{-2}$ & $(0.2) 2.8(7.2) \times 10^{-16}$ & $(0.00) 0.65(0.67)$ & $>26$ \\
Soot & $\mathrm{C}^{5+}$ & 50 & 2.864 & $(0.9) 8.3(19) \times 10^{-15}$ & $(0.00) 0.64(0.68)$ & $>26$ \\
Soot & $\mathrm{Si}^{7+}$ & 85 & 13.7 & $(1.6) 4.2(6.8) \times 10^{-14}$ & $(0.16) 0.31(0.33)$ & $>55$ \\
Soot & $\mathrm{Ni}^{9+}$ & 100 & 39.35 & $(0.9) 1.9(3.3) \times 10^{-13}$ & $(0.31) 0.39(0.40)$ & $>45$ \\
\hline
\end{tabular}

Notes. The aliphatic C-H destruction cross section $\sigma_{\mathrm{d}}$, the relative asymptotic optical depth $A_{\mathrm{f}} / A_{\mathrm{i}}$, and $1 / \rho_{\mathrm{f}}$ are given (see text for explanation). The extreme values of each parameter are given between parenthesis.

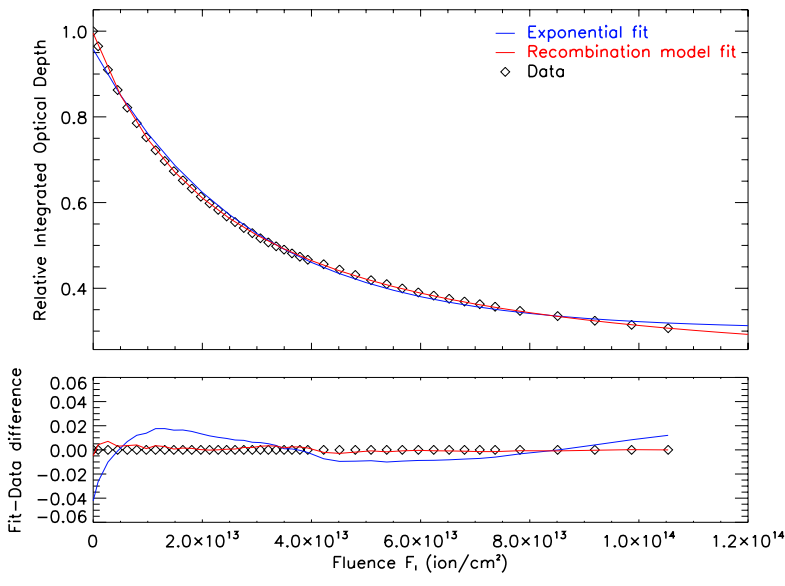

Fig. B.1. Comparison between the two different best fits of the hydrogen release induced by ion irradiation. This example corresponds to irradiation by $85 \mathrm{MeV} \mathrm{Si}^{7+}$.

\section{Appendix B: Comparison of the recombination model with the exponential fit}

We performed the two kinds of fit with our data, based on Eqs. (2) and (A.1), using $\sigma_{\mathrm{d}}$ and $\rho_{\mathrm{f}}$ as adjustable parameters. For all experiments, the best fit based on the bulk molecular recombination (Eq. (2)) has a value of $\chi^{2}$ lower or equal to the one corresponding to the best fit using Eq. (A.1). Therefore, even if the fits using Eq. (A.1) agree well with the experimental data, Eq. (2) provides a better representation of hydrogen concentration in the irradiated films than Eq. (A.1) (see a comparison of the two different best fits determined with each method in Fig. B.1). This is why we use the recombination model results in this article.

The uncertainties on the hydrogen release cross section determined by Eq. (2) are greater than those determined with Eq. (A.1) because $\sigma_{\mathrm{d}}$ is more dependent on the value of $\rho_{\mathrm{f}}$ in Eq. (2) than in Eq. (A.1). To reduce these uncertainties, it would be necessary to determine the asymptotic behaviour more precisely and thus irradiate with fluxes that are too high (very far
Table C.2. Values of the $\sigma_{\mathrm{d}}$ fit parameters from Eq. (5).

\begin{tabular}{ccc}
\hline \hline Material & $\alpha$ & $K$ \\
\hline a-C $: \mathrm{H}$ & $1.3( \pm 0.3)$ & $1.0( \pm 0.4) 10^{-15}$ \\
Soot & $1.0( \pm 0.3)$ & $4.0( \pm 2.3) 10^{-15}$ \\
\hline
\end{tabular}

Notes. $K$ is given in $\mathrm{cm}^{2-2 \alpha} \mathrm{MeV}^{-\alpha} \mathrm{mg}^{\alpha}$ units. $\sigma_{\mathrm{d}}$ are obtained from the exponential fits.

from the interstellar one, and that induce heating of the sample) or during times that are far longer. Such a long time was not possible to reach within the available time on the ion irradiation facility.

\section{Appendix C: Aliphatic C-H destruction parameters determined by the exponential fit}

The obtained destruction cross section $\sigma_{\mathrm{d}}$, relative final hydrogen content $A_{\mathrm{f}} / A_{\mathrm{i}}$, and corresponding volume $1 / \rho_{\mathrm{f}}$ values obtained for the different experiments with the exponential fits are reported in Table C.1. The obtained $\sigma_{\mathrm{d}}$ values are plotted as a function of $S_{\mathrm{e}}$ in Fig. C.1, for a-C:H and soot samples. As with the recombination model, $\sigma_{\mathrm{d}}$ increases with $S_{\mathrm{e}}$ as a power law, and this correlation can be fitted by Eq. (5). The corresponding values of $\alpha$ and $K$ are given in Table C.2 for a-C:H and soot. The $\sigma_{\mathrm{d}}$ values obtained with the exponential fit are slightly higher than those obtained with the recombination model, but the $\alpha$ possible values obtained with the exponential fit agree with those obtained with the recombination model.

In Fig. C.1 we also represented $\sigma_{\mathrm{d}}$ values obtained with the exponential fit for a-C:H from data found in the literature (Adel et al. 1989; Baptista et al. 2004; Baumann et al. 1987; Fujimoto et al. 1988; González-Hernández et al. 1988; Ingram \& McCormick 1988; Marée et al. 1996; Mennella et al. 2003; Pawlak et al. 1997; Prawer et al. 1987; Som et al. 2005, 1999; Zou et al. 1988). In the article of Pawlak et al. (1997), many $\sigma_{\mathrm{d}}$ have been calculated with exponential fits, but data of 


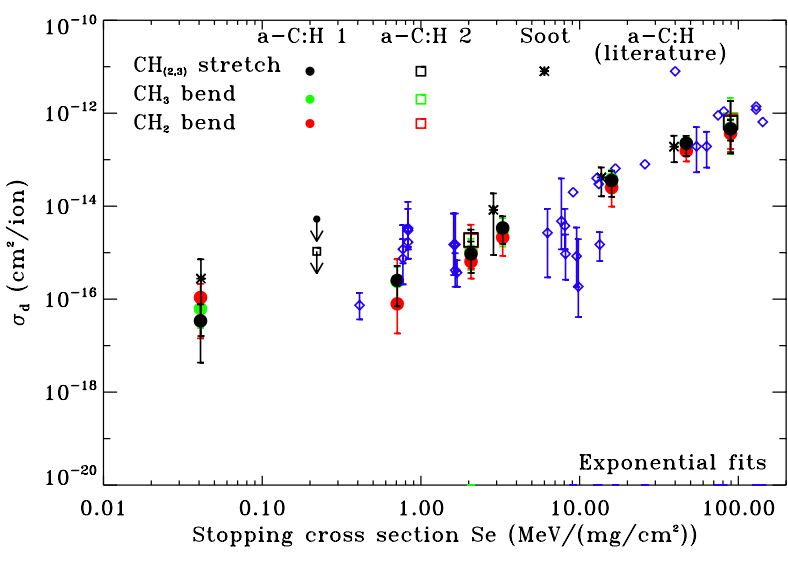

Fig. C.1. Aliphatic C-H destruction cross section $\sigma_{\mathrm{d}}$ obtained with the exponential fit as a function of the electronic cross section $S_{\mathrm{e}}$ for the different ion/energy irradiation of our a-C:H (filled dots for a-C:H 1 , and squares for a-C:H 2) and soot (black asterisks) samples. Cross sections calculated from data of different a-C:H irradiation studies are represented with blue diamonds (Adel et al. 1989; Baptista et al. 2004; Baumann et al. 1987; Fujimoto et al. 1988; González-Hernández et al. 1988; Ingram \& McCormick 1988; Marée et al. 1996; Mennella et al. 2003; Pawlak et al. 1997; Prawer et al. 1987; Som et al. 2005, 1999; Zou et al. 1988). See text for details.

the hydrogen depletion as a function of the fluence does not appear for most of these experiments. These data appear thus without error bars in Fig. C.1. As in Fig. 4, we did not plot results with $S_{\mathrm{n}} / S_{\mathrm{e}}>0.5$, or $d / R_{\mathrm{p}}>0.9$.

\section{References}

Adamson, A. J., Whittet, D. C. B., \& Duley, W. W. 1990, MNRAS, 243, 400 Adel, M. E., Amir, O., Kalish, R., \& Feldman, L. C. 1989, J. Appl. Phys., 66, 3248

Allamandola, L. J., Sandford, S. A., Tielens, A. G. G. M., \& Herbst, T. M. 1993, Science, 260, 64

Baptista, D. L., Garcia, I. T. S., \& Zawislak, F. C. 2004, Nucl. Instrum. Methods Phys. Res. B, 219, 846

Baumann, H., Rupp, T., Bethge, K., Koidl, P., \& Wild, C. 1987, Eur. Mater. Res. Soc. Conf. Proc., 17, 343

Bond, T. C., \& Bergstrom, R. W. 2006, Aerosol Science and Technology, 40, 27 Bottinelli, S., Adwin Boogert, A. C., Bouwman, J., et al. 2010, ApJ, 718, 1100 Brooke, T. Y., Sellgren, K., \& Geballe, T. R. 1999, ApJ, 517, 883

Brunetto, R., Baratta, G. A., \& Strazzulla, G. 2004, J. Appl. Phys., 96, 380

Brunetto, R., Pino, T., Dartois, E., et al. 2009, Icarus, 200, 323

Chiar, J. E., Adamson, A. J., \& Whittet, D. C. B. 1996, ApJ, 472, 665

Chiar, J. E., Tielens, A. G. G. M., Whittet, D. C. B., et al. 2000, ApJ, 537, 749

Cravens, T. E., \& Dalgarno, A. 1978, ApJ, 219, 750

Dalgarno, A. 2006, Proceedings of the National Academy of Science, 103, 12269

Dartois, E., \& d'Hendecourt, L. 2001, A\&A, 365, 144

Dartois, E., d'Hendecourt, L., Thi, W., Pontoppidan, K. M., \& van Dishoeck, E. F. 2002, A\&A, 394, 1057

Dartois, E., Muñoz Caro, G. M., Deboffle, D., Montagnac, G., \& D’Hendecourt, L. 2005, A\&A, 432, 895

Dartois, E., Geballe, T. R., Pino, T., et al. 2007, A\&A, 463, 635

Draine, B. T. 1990, in The Evolution of the Interstellar Medium, ed. L. Blitz, ASP Conf. Ser., 12, 193

Elmegreen, B. G. 2007, ApJ, 668, 1064

Fujimoto, F., Tanaka, M., Iwata, Y., et al. 1988, Nucl. Instrum. Methods Phys. Res. B, 33, 792

Glover, S. C. O., \& Mac Low, M. 2007, ApJ, 659, 1317

Godard, M., \& Dartois, E. 2010, A\&A, 519, A39
Goldsmith, P. F., Li, D., \& Krčo, M. 2007, ApJ, 654, 273

González-Hernández, J., Asomoza, R., Reyes-Mena, A., et al. 1988, J. Vacuum Sci. Techn., 6, 1798

Hörandel, J. R. 2008, Adv. Space Res., 41, 442

Indriolo, N., Geballe, T. R., Oka, T., \& McCall, B. J. 2007, ApJ, 671, 1736

Indriolo, N., Fields, B. D., \& McCall, B. J. 2009, ApJ, 694, 257

Ingram, D. C., \& McCormick, A. W. 1988, Nucl. Instrum. Methods Phys. Res. B, 34, 68

Jones, A. P., Tielens, A. G. G. M., Hollenbach, D. J., \& McKee, C. F. 1994, ApJ, 433, 797

Le Petit, F., Nehmé, C., Le Bourlot, J., \& Roueff, E. 2006, ApJS, 164, 506

Marée, C., Vredenberg, A., \& Habraken, F. 1996, Mater. Chem. Phys., 46, 198

Mathis, J. S., Mezger, P. G., \& Panagia, N. 1983, A\&A, 128, 212

McCall, B. J., Huneycutt, A. J., Saykally, R. J., et al. 2003, Nature, 422, 500

McKee, C. 1989, in Interstellar Dust, ed. L. J. Allamandola, \& A. G. G. M. Tielens, IAU Symp., 135, 431

Mennella, V. 2006, ApJ, 647, L49

Mennella, V. 2008, ApJ, 682, L101

Mennella, V. 2010, ApJ, 718, 867

Mennella, V., Brucato, J. R., Colangeli, L., \& Palumbo, P. 1999, ApJ, 524, L71

Mennella, V., Muñoz Caro, G. M., Ruiterkamp, R., et al. 2001, A\&A, 367, 355

Mennella, V., Brucato, J. R., Colangeli, L., \& Palumbo, P. 2002, ApJ, 569, 531

Mennella, V., Baratta, G. A., Esposito, A., Ferini, G., \& Pendleton, Y. J. 2003, ApJ, 587, 727

Meyer, J., Drury, L. O., \& Ellison, D. C. 1998, Space Sci. Rev., 86, 179

Möller, W., \& Scherzer, B. M. U. 1987, Appl. Phys. Lett., 50, 1870

Moskalenko, I. V., Strong, A. W., Ormes, J. F., \& Potgieter, M. S. 2002, ApJ, 565,280

Mouschovias, T. C., Tassis, K., \& Kunz, M. W. 2006, ApJ, 646, 1043

Muñoz Caro, G. M., Ruiterkamp, R., Schutte, W. A., Greenberg, J. M., \& Mennella, V. 2001, A\&A, 367, 347

Murakawa, K., Tamura, M., \& Nagata, T. 2000, ApJS, 128, 603

Padovani, M., Galli, D., \& Glassgold, A. E. 2009, A\&A, 501, 619

Pawlak, F., Paumier, E., Toulemonde, M., et al. 1997, Nucl. Instrum. Methods Phys. Res. B, 131, 135

Pendleton, Y. J., \& Allamandola, L. J. 2002, ApJS, 138, 75

Pendleton, Y. J., Sandford, S. A., Allamandola, L. J., Tielens, A. G. G. M., \& Sellgren, K. 1994, ApJ, 437, 683

Pino, T., Dartois, E., Cao, A.-T., et al. 2008, A\&A, 490, 665

Prasad, S. S., \& Tarafdar, S. P. 1983, ApJ, 267, 603

Prawer, S., Kalish, R., Adel, M., \& Richter, V. 1987, J. Appl. Phys., 61, 4492

Sandford, S. A., Allamandola, L. J., Tielens, A. G. G. M., et al. 1991, ApJ, 371, 607

Shen, C. J., Greenberg, J. M., Schutte, W. A., \& van Dishoeck, E. F. 2004, A\&A, 415,203

Simpson, J. A. 1983, Ann. Rev. Nucl. Part. Sci., 33, 323

Smith, R. G., Sellgren, K., \& Brooke, T. Y. 1993, MNRAS, 263, 749

Som, T., Kumar, S., \& Kulkarni, V. N. 1999, Nucl. Instrum. Methods Phys. Res. B, 156,212

Som, T., Malhotra, M., Kulkarni, V. N., \& Kumar, S. 2005, Physica B Condensed Matter, 355, 72

Sorrell, W. H. 1990, MNRAS, 243, 570

Spitzer, Jr., L., \& Tomasko, M. G. 1968, ApJ, 152, 971

Spoon, H. W. W., Armus, L., Cami, J., et al. 2004, ApJS, 154, 184

Strazzulla, G., Baratta, G. A., Leto, G., Palumbo, M. E., \& Spinella, F. 2001, in Exo-/Astro-Biology, ed. P. Ehrenfreund, O. Angerer, \& B. Battrick, ESA SP, 496, 129

Tassis, K., \& Mouschovias, T. C. 2004, ApJ, 616, 283

Vladimirov, A., Digel, S., Jóhannesson, G., et al. 2011, Comput. Phys. Commun., 182, 1156

Webber, W. R. 1998, ApJ, 506, 329

Webber, W. R., \& Yushak, S. M. 1983, ApJ, 275, 391

Whittet, D. C. B., Bode, M. F., Longmore, A. J., et al. 1988, MNRAS, 233, 321

Whittet, D. C. B., Boogert, A. C. A., Gerakines, P. A., et al. 1997, ApJ, 490, 729

Wickramasinghe, D. T., \& Allen, D. A. 1980, Nature, 287, 518

Wild, C., \& Koidl, P. 1987, Appl. Phys. Lett., 51, 1506

Ziegler, J. F., Ziegler, M. D., \& Biersack, J. P. 2010, Nucl. Instrum. Methods Phys. Res. B, 268, 1818

Zou, J. W., Schmidt, K., Reichelt, K., \& Stritzker, B. 1988, J. Vacuum Sci. Techn., 6, 3103 